\title{
Individual differences show that only some bats can cope with noise-induced masking and distraction
}

\author{
Dylan G.E. Gomes ${ }^{\text {Corresp., } 1,2 \text {, Holger R. Goerlitz }}{ }^{1}$ \\ ${ }^{1}$ Max Plank Institute for Ornithology, Seewiesen, Bavaria, Germany \\ 2 Department of Biological Sciences, Boise State University, Boise, Idaho, United States \\ Corresponding Author: Dylan G.E. Gomes \\ Email address: dylangomes@u.boisestate.edu
}

Anthropogenic noise is a widespread pollutant that has received considerable recent attention. While alarming effects on wildlife have been documented, we have limited understanding of the perceptual mechanisms of noise disturbance, which are required to understand potential mitigation measures. Likewise, individual differences in response to noise (especially via perceptual mechanisms) are likely widespread, but lacking in empirical data. Here we use the echolocating bat Phyllostomus discolor, a trained discrimination task, and experimental noise playback to explicitly test perceptual mechanisms of noise disturbance. We demonstrate high individual variability in response to noise treatments and evidence for multiple perceptual mechanisms. Additionally, we highlight that only some individuals were able to cope with noise, while others were not. We tested for changes in echolocation call duration, amplitude, and peak frequency as possible ways of coping with noise. Although all bats strongly increased call amplitude and showed additional minor changes in call duration and frequency, these changes could not explain the differences in coping and non-coping individuals. Our understanding of noise disturbance needs to become more mechanistic and individualistic as research knowledge is transformed into policy changes and conservation action. 
1 Individual differences show that only some bats can cope with noise-induced masking and distraction

5 Authors: Dylan G.E. Gomes ${ }^{1,2, *}$ \& Holger R. Goerlitz ${ }^{1}$

$8{ }^{1}$ Acoustic and Functional Ecology, Max Plank Institute for Ornithology, Seewiesen, Germany

92 Present address: Boise State University, Boise, Idaho, USA, 83725-1515

$10 *$ Correspondence: dylangomes@u.boisestate.edu

Keywords: anthropogenic noise, echolocation, perceptual mechanism, Chiroptera,

Phyllostomidae, discrimination task

We dedicate this research to Lutz Wiegrebe, who passed away in November 2019. Lutz was a great friend and a brilliant scientist with a sharp mind. We will miss his thoughts and jokes forever. 
22 Abstract

23 Anthropogenic noise is a widespread pollutant that has received considerable recent attention.

While alarming effects on wildlife have been documented, we have limited understanding of the perceptual mechanisms of noise disturbance, which are required to understand potential mitigation measures. Likewise, individual differences in response to noise (especially via perceptual mechanisms) are likely widespread, but lacking in empirical data. Here we use the echolocating bat Phyllostomus discolor, a trained discrimination task, and experimental noise playback to explicitly test perceptual mechanisms of noise disturbance. We demonstrate high individual variability in response to noise treatments and evidence for multiple perceptual mechanisms. Additionally, we highlight that only some individuals were able to cope with noise, while others were not. We tested for changes in echolocation call duration, amplitude, and peak frequency as possible ways of coping with noise. Although all bats strongly increased call amplitude and showed additional minor changes in call duration and frequency, these changes could not explain the differences in coping and non-coping individuals. Our understanding of noise disturbance needs to become more mechanistic and individualistic as research knowledge is transformed into policy changes and conservation action.

\section{Introduction}

Anthropogenic noise is a global pollutant that is pervasive even in protected areas (Buxton et al., 2017), is increasing over time (Luther and Baptista, 2009), and has recently gained considerable attention by behavioral biologists (Barber et al., 2010). Noise can disrupt animal behavior, such as communication (Brumm and Slabbekoorn, 2005; Rabin et al., 2003) and foraging (Gomes et al., 2016; Purser and Radford, 2011; Siemers and Schaub, 2011), reduce reproductive success (Halfwerk et al., 2011), increase mortality (Simpson et al., 2016), change biological communities (Francis et al., 2011), and alter ecological services (Francis et al., 2012). Yet it is not often understood what mechanisms drive these changes, and if and how different individuals are affected by these mechanisms differently. Individual differences in response to noise has been documented in humans (Furnham and Strbac, 2002; Standing et al., 1990), birds (Naguib et al., 2013), fish (Bruintjes and Radford, 2013), mongooses (Eastcott et al., 2020), and bats (Luo et al., 
52 2019), yet individual differences are often overlooked as individuals are more often grouped 53 together for analysis than analyzed individually (Harding et al., 2019).

54 Similarly, only a few studies to date have investigated the mechanisms of noise disturbance.

55 Some have used bands of noise that are both overlapping or non-overlapping with signals or cues

56 of interest to demonstrate evidence of masking (e.g. Gomes et al., 2016; Zhou et al., 2019).

57 Others have shown that noise can disrupt behaviors across sensory modalities (where masking cannot work) via distraction (e.g. Chan et al., 2010; Morris-Drake et al., 2016). Understanding how we may be able to mitigate the consequences of noise relies heavily on knowledge of direct mechanisms of noise disturbance on individuals. Dominoni et al. (2020), for example, highlight three main perceptual mechanisms of noise disturbance - masking, distraction, and misleading. While these mechanisms apply to all senses, we here consider them specifically in the auditory domain.

Masking is a mechanism whereby noise overlaps in frequency with important signals or cues, thus making the detection and auditory analysis of the signal difficult, if not impossible, and occurs at the auditory periphery (Clark et al., 2009; Fay and Wilber, 1989; Gomes et al., 2016; Tanner Jr, 1958). Distraction, on the other hand, occurs when noise competes for the finite attention (i.e. central processing) of an organism, and is not limited to frequencies that overlap with a signal or cue of interest (Chan et al., 2010). Misleading occurs when noise is interpreted as something that it is not, similar to a false alarm (Wiley, 2013) or a sensory trap (Christy, 1995; West-Eberhard, 1984). Some have shown, for example, that beaked whales respond to Navy sonar similarly as they do killer whales, perhaps indicating that they misinterpret this noise as a predator or something unknown that might be dangerous (Tyack et al., 2011). Other mechanisms of disturbance have been proposed, such as stress, fear, and avoidance (Campo et al., 2005; Luo et al., 2015a; Voellmy et al., 2014), yet these physiological and behavioral responses must occur downstream of the initial perceptual mechanism (i.e. masking, distraction, or misleading).

Here, we use a behavioral experiment to tease apart the effects of both masking and distraction as perceptual mechanisms on individual echolocating bats. Echolocating bats are a worthwhile system to study these questions because they actively sense their world via sound. Thus, by 
82 monitoring how they adjust the characteristics of their echolocation calls in response to noise, we 83 can easily study how they are responding to changes in the sensory environment.

84 Anthropogenic noise arises from diverse sources (e.g. automobile and airplane traffic, energy extraction, and urban cities) and generally contains more energy in lower rather than in higher frequencies (Bondello and Brattstrom, 1978; Bunkley and Barber, 2015; Bunkley et al., 2015; Cinto Mejia et al., 2019; Nemeth and Brumm, 2010; Schaub et al., 2008; Siemers and Schaub, 2011; Sierro et al., 2017). Despite this, anthropogenic noise can contain considerable energy in the ultrasonic range, particularly at close distance to the noise source (Siemers and Schaub, 2011). As the echolocation call frequencies of different bat species also span a wide frequency range (Fenton and Bell, 1981), anthropogenic noise can overlap bat echolocation in frequency in a species-, distance- and noise-source specific manner, potentially affecting bats via multiple mechanisms (Dominoni et al., 2020).

We trained pale spear-nosed bats (Phyllostomus discolor) to discriminate surface structures with increasing level of difficulty and under three noise treatments (see Methods). We made distinct predictions for each of the tested perceptual mechanisms. By broadcasting noise that does and does not spectrally overlap with echolocation calls, we directly tested the role of masking. We predicted that masking should only reduce the discrimination performance for spectrally overlapping noise, but not for non-overlapping noise. Since distraction assumes that deficits result from limited attentional resources, we predicted that distraction should be independent of the noises' spectral overlap with echolocation calls (distinguishing it from masking), but should depend on the noises' temporal structure. We thus also presented a spectrally overlapping 'sparse' noise with random temporal gaps, making the noise less predictable, and thus, more distracting (Glass and Singer, 1972; Kjellberg et al., 1996; Matthews et al., 1980). At the same time, sparse noise might allow bats to listen in-between the noise gaps ("dip listening"), reducing its masking effect (Vélez and Bee, 2011). Thus, if distraction is the primary mechanism of disturbance, then sparse noise should decrease discrimination performance and increase trial duration, while we would expect an increase in discrimination performance and a decrease in trial duration under sparse noise if masking is the primary mechanism of disturbance. 


\section{Materials and methods}

114

\section{Experimental Animals and Husbandry}

116 The pale spear-nosed bat (Phyllostomus discolor; Wagner, 1843) is an omnivorous neotropical

117 bat (Kwiecinski, 2006) that emits multi-harmonic, downward frequency-modulated echolocation 118 calls of short duration $(0.3-2.5 \mathrm{~ms}$ ) and most energy in the range of $\sim 40-100 \mathrm{kHz}$ (Goerlitz et

119 al., 2008; Kwiecinski, 2006). A captive colony of $P$. discolor was kept in a temperature $\left(\sim 25^{\circ} \mathrm{C}\right)$ 120 and humidity ( 70\%) controlled room at the Max Planck Institute for Ornithology, Seewiesen, 121 Germany, where the bats had access to water ad libitum, and were fed a fruit-based diet. During 122 experimental days, bats were first only fed during experiments (mealworm reward; see below), to 123 maintain motivation. At the end of the day, several hours later, bats were fed fruit. Experiments 124 were carried out in a nearby, but separate room $\left(\sim 21{ }^{\circ} \mathrm{C} / 65 \%\right.$ rel. hum $)$. Bat housing and all 125 research was approved by the German authorities under the permit numbers 311.5-5682.1/1126 2014-023 (Landratsamt Starnberg) and 55.2-1-54-2532-18-15 (Regierung von Oberbayern), 127 respectively.

\section{Experimental Setup}

130 Experiments were conducted in a dark chamber within a dark room (see below for light levels).

131 Walls of both the chamber and the room were covered in anechoic foam to reduce echoes. The

132 chamber held a custom-built mushroom maze (87 cm x $65 \mathrm{~cm}$ x $18 \mathrm{~cm}, \mathrm{~W} \times \mathrm{H}$ x D), which was a 133 fully enclosed mesh-cage (Baier et al., 2019). The mesh (12 mm x $12 \mathrm{~mm}$ mesh grid with wires of $<1 \mathrm{~mm}$ diameter) is acoustically transparent for the echolocation calls of $\mathrm{P}$. discolor (wavelength: $\sim 3-9 \mathrm{~mm}$ ). This maze allowed the bats perceptual access by echolocation to two simultaneously presented stimulus discs (reference plus test disc) on either side of the maze

(Figure 1A). One infrared light barrier next to each of the disc positions objectively recorded the choice of the bat via a custom-written Matlab code (The Mathworks, Nattick, MA, USA), avoiding observer bias and potential observer errors. Two loudspeakers (Vifa, Peerless by Tymphany, San Rafel, CA, USA; connected to power amplifier TA-FE330R, Sony, Tokyo, Japan, and soundcard Fireface 802, RME, Haimhausen, Germany) were mounted on either side

142 of the setup for noise playback (Figure 1A). The experimenter (stationed outside of the 143 chamber) observed the experiment via a red-filtered computer screen displaying a live-feed from 
144 an infrared camera (Foculus FO432SB; NET-GmbH, Finning, Germany; 880 nm infrared LED-

145 illumination, TV6818; ABUS, Wetter, Germany).

146

\section{Stimuli}

148 We used an established behavioral assay that has been previously used to test perceptual performance in bats (Baier et al., 2019). We used eight discs with $45 \mathrm{~cm}$ diameter as physical stimuli. The stimulus discs were made by a milling cutter (Modellbau Grossmann, Calw,

151 Germany) and then spray-painted with multiple coats to be smooth-textured. One disc

152 ("reference disc") had a completely flat surface. The seven other discs had concentric ripples,

153

154

155

156

157

158

159

160

161

162

163

164

165

166

167

168

169

170

171

172

173

174 resembling concentric sinusoidal standing waves. All rippled discs had the same spatial frequency of 17.8 ripples per meter, corresponding to eight full sinusoidal ripples per disc, but different ripple heights increasing from 2 to $32 \mathrm{~mm}$ peak-to-peak height $(2,4,5.6,8,11.2,16,32$ $\mathrm{mm}$; Figure 1B).

\section{Noise Treatments}

In addition to silence, used as a control, we tested the bats under three band-limited white noise treatments (Figure 2): 1) Smooth non-overlapping noise: band-limited white Gaussian noise not overlapping in frequency with the echolocation calls of $P$. discolor, ranging from $5-35 \mathrm{kHz}\left(10^{\text {th }}\right.$ order Butterworth filter). 2) Smooth-overlapping noise: band-limited white Gaussian noise overlapping in frequency with the echolocation calls of $P$. discolor, ranging from $40-90 \mathrm{kHz}$ (10 ${ }^{\text {th }}$-order Butterworth filter). 3) Sparse-overlapping noise: derived from the smoothoverlapping noise $(40-90 \mathrm{kHz})$ with additional short silent gaps between all adjacent samples (Hübner and Wiegrebe, 2003). These silent gaps generate fluctuations in the temporal envelope of the noise, causing the noise to sound rougher in comparison to the smooth noise. The duration of the silent gaps was drawn from a uniformly random duration (mean: 0.3 ms, range: 0-0.6 ms). The roughness is quantified by the base-10 logarithm of the waveform's fourth moment (Hartmann and Pumplin, 1988), and is calculated as the summed amplitude values raised to the power of 4, divided the squared sum of all squared amplitude values raised to the power of 2 . LogM4 was $1.44 \log$ M4 for the sparse-overlapping noise, compared to $0.48 \log \mathrm{M} 4$ for the two smooth noises (cf. Grunwald et al., 2004). For all three noise types, we generated uncorrelated stereo noise files of $60 \mathrm{~min}$ duration (192 kHz sampling rate, 16 bit resolution) and corrected 
175

176

177

178

179

180

181

182

183

184

185

186

187

188

189

190

191

192

193

194

195

196

197

198

199

200

201

202

203

204 205

each channel for the corresponding speaker's frequency response. At each daily experimental session, noise playback was started at least $30 \mathrm{~s}$ prior to the beginning of the first trial at a random position within the 60-min long noise files, and continued throughout the session.

Playback level was $70 \mathrm{~dB}$ SPL RMS re. $20 \mu \mathrm{Pa}$ at the bat's starting position. It is important to note that the perceived loudness was likely different, since the noise treatments had different bandwidths and the auditory sensitivity of $P$. discolor varies over their range of hearing (Esser and Daucher, 1996; Hoffmann et al., 2008). However, this should not affect any interpretation of the designed tests of masking and distraction.

\section{Training and testing}

Nine bats were initially trained in a two-alternative forced-choice paradigm to discriminate the flat reference disc from the stimulus disc with the highest ripples $(32 \mathrm{~mm})$. During training, bats received mealworms (larvae of Tenebrio molitor) as reward when approaching the flat reference disc only. Two bats did not learn this task at all, and three bats appeared to learn somewhat but never showed consistent performance after three months of training (including one bat that had already been trained successfully in a previous experiment; Baier et al., 2019). There appears to be no consistent patterns between failure to train and previous experience or age of the bats. Only four bats ( 3 males, 1 female) reached the training criterion ( $>70 \%$ correct approaches to the flat reference disc over three consecutive days; the three males were previously trained in Baier et al., 2019) and were subsequently tested during the data acquisition stage. Throughout testing and training, the flat reference disc was pseudo-randomly (Gellermann, 1933) alternated between each side of the experimental setup to avoid any biases in location preferences by the bats.

Prior to each trial, bats were encouraged to enter a small Tupperware 'starting' box in the middle of the experimental setup by offering blended banana as a food reward via a syringe tube that was mounted inside this starting box (Figure 1A). While in the starting box, bats had no perceptual access to the discs since the solid bottom door was closed upon entering. The discs were then placed in their positions. Once the starting box opened, the trial started. Bats were allowed to crawl through the setup towards the discs. When they broke the IR light barrier, the trial ended, and the bat was rewarded with a mealworm when it chose the flat reference disc. 
206 Bats were initially tested in a silent (i.e. ambient sound level) experimental room to generate

207 baseline psychometric curves with discs of 5 different ripple heights $(2 \mathrm{~mm}, 4 \mathrm{~mm}, 8 \mathrm{~mm}, 16$

$208 \mathrm{~mm}$, and $32 \mathrm{~mm}$ ). For the subsequent tests, we added two additional discs with intermediate

209 ripple heights $(5.6 \mathrm{~mm}$ and $11.2 \mathrm{~mm})$ to get better resolution around the turning point of the

210 psychometric function measured in silence. The bats were then tested with all seven discs in each

211 of the three noise conditions. Per individual, the noise conditions were presented in pseudo-

212 random sequence, and a new noise condition was presented only after the individual had

213 completed all trials of the previous noise condition. Finally, each bat was retested a second time

214 in silence with all seven discs, to assure that differences in performance were not due to learning

215 or other order effects. Testing each bat in the same noise conditions over multiple trials and days

216 (as opposed to changing noise treatment for each trial) may lead to more habituation to that noise

217 treatment. However, as the opportunity for habituation was consistent across treatments, any

218 differences amongst noise treatments are due to the noise treatments themselves. Additionally, as

219 randomizing noise treatments across trials would only exacerbate any differences amongst noise

220 treatments, the differences we report here are conservative. Each bat was tested 30 times for

221 every ripple height and noise treatment combination, totaling 990 trials per individual bat.

222

223 To motivate the bats, each day was started with easier discrimination tasks (higher ripple

224 heights) and gradually moved towards more difficult tasks (lower ripple heights). Bats were

225 allowed to continue testing until satiated or no longer food-motivated, which was determined by

226 the bat attempting to leave the mushroom maze via an exit door in the top of the setup.

227

228

\section{Aborted trials}

229 If the bat did not exit the starting box within 5 minutes after starting a trial, the trial was aborted 230 and repeated. As the bats did not make a decision in those aborted trials, they were not included

231 in further analyses. The one exception, however, is that we analyzed the number of these aborted

232 trials as a measure of aversion to the noise. Abortion was behaviorally distinguished from

233 satiation, as bats would crawl toward the door to exit the maze when they were seemingly no

234 longer food-motivated. It is likely that bats aborted trials not only because of the noise, but also

235 for potential other reasons. However, as those other reasons are constant within the controlled 
236 setting of an experiment, any difference in the number of aborted trials (as in any other

237 behavioral measure) can be attributed to the experimental treatment (i.e., the type of noise).

238

239

240

\section{Echolocation call recording and analysis}

We continuously recorded the bats' echolocation calls into a 4-sec-long ring-buffer. When a bat

241 made a decision by interrupting a light barrier, only the sound of the four seconds prior to the decision were automatically saved into a WAV-file. Recordings were performed with two microphones (Knowles SPU0410) positioned just behind each light barrier, a sound card (Fireface 802, RME, Haimhausen, Germany; $192 \mathrm{kHz}$ sampling rate, 16-bit resolution) and playrec (V2.1.0, playrec.co.uk) for Matlab (V2007b, The Mathworks, Nattick, MA, USA).

Echolocation calls were analyzed automatically by custom-written scripts in Matlab (V2016a, The Mathworks, Nattick, MA, USA), advanced from previous work (Goerlitz et al., 2008; Luo et al., 2015a). First, we filtered all recordings with each microphone's compensatory impulse response (511-order finite impulse response filter) to compensate for the microphone's frequency response, and a band-pass filter $(38-95 \mathrm{kHz}, 8$ th-order elliptic filter). Second, we used a threshold detector to broadly determine the timing of all acoustic events: we additionally bandpass-filtered recordings around the bats' main call energy $(45-90 \mathrm{kHz}$, 4th-order elliptic filter), calculated their low-pass filtered $(500 \mathrm{~Hz}$, 4th-order elliptic filter) Hilbert-envelope, and detected all acoustic events that surpassed a threshold (mean $+2 x$ STD of the envelope), excluding events that were too close to the preceding event $(<20 \mathrm{~ms})$ and too short $(<0.75 \mathrm{~ms})$. We then added an additional $0.5 \mathrm{~ms}$ on both sides of the detected acoustic events, which, together with the previous low-pass-filtering of the envelope, ensured that the determined time window included the full call flanked by non-call samples. Third, we detected the actual call within this time window of the recording, and analyzed its acoustic parameters.

261

262 Call duration was determined from the low-pass filtered $(5000 \mathrm{~Hz}, 2$ nd-order butterworth filter) Hilbert envelope of the originally filtered recording $(38-95 \mathrm{kHz})$ at $-12 \mathrm{~dB}$ below the envelope's peak value. Peak frequency (frequency with highest amplitude), frequency centroid (dividing the call energy into two halves along the frequency axis; Au, 2012) and the lowest and highest 
267 the highest amplitude) were calculated from the time-averaged call spectrogram (1024 FFT of

268100 samples, 95\% overlap). Relative call level was calculated as the root mean square (RMS) of

269 all samples within the $-12 \mathrm{~dB}$ duration criterion and expressed in $\mathrm{dB} F S$, i.e., negative $\mathrm{dB}$ values

270 relative to the full scale of the recording system.

271

272 If a call was detected on both microphones, we only analyzed the call with the higher signal-to-

273 noise-ratio (SNR: call-RMS relative to RMS of all parts of the recording that were not classified

274 as acoustic events). Of all recorded calls $(\mathrm{N}=287,061)$, we excluded for further analysis calls

275 shorter than $0.3 \mathrm{~ms}$ and longer than $2 \mathrm{~ms}$, with too high $(>-0.5 \mathrm{~dB} F S$, to avoid clipping) or too

276 low recorded peak amplitudes (<-15 dB FS), with a SNR of less than $20 \mathrm{~dB}$, and whose ratio

277 between the $-12 \mathrm{~dB}$ duration and the $-6 \mathrm{~dB}$ duration was larger than 1.5 (to exclude calls with

278 long echoes). All remaining calls $(\mathrm{N}=63,990)$ were manually viewed as spectrogram (256 FFT,

27950 time slices over full call length, 95\% overlap), blind to bat individual and noise treatment, to

280 exclude ambiguous recordings and obvious artefacts, e.g., overlapping call-echo-pairs and non-

281 multiharmonic sounds (e.g., clicks, external noise). This resulted in a final data set of 59,173

282 calls ( $0-83$ calls per trial). Of the 3960 total trials, 3469 trials $(87.6 \%)$ included at least one

283 recorded call, 3166 trials (80.0\%) at least 3 calls, 2912 trials $(73.5 \%)$ at least 5 calls, and 2265

284 trials $(57.2 \%)$ included at least 10 recorded calls. For further analysis, we used the mean call

285 parameters of each trial in statistical models. Note that the background noise affects our call level

286 measurements only negligibly $(<0.05 \mathrm{~dB})$ because we only analyzed calls with a SNR $>20 \mathrm{~dB}$.

\section{Visual system and light levels}

289 Light levels in the experimental room were extremely low $\left(1.39 \times 10^{-5}\right.$ lux; SPM068 with

290 ILT1700 light detector, resolution 10-7 lux, International Light Technologies, Peabody, MA,

291 USA), precluding the use of vision to discriminate between discs. Many other laboratory

292 experiments, which have similarly excluded the use of vision due to an assumed unavailability of

293 light, have either reported higher light levels than us or did not measure or report light levels.

294 Additionally, it has been experimentally shown that another related phyllostomid bat (Macrotus

295 californicus) only has visual acuity to light levels as low as $2 \times 10^{-3}$ lux (Bell and Fenton, 1986),

296 which is nearly two orders of magnitude higher than our light levels. Furthermore, $M$.

297 californicus has one of the highest sensitivities to low light levels known (Bell and Fenton, 1986; 
298 Eklöf et al., 2014). Thus, it is extremely unlikely that the Phyllostomus discolor used here were 299 able to visually discriminate between the discs.

300

301

302

303

\section{Statistical analysis}

We fitted (generalized) linear models to the behavioral data of each individual, using R (R Core Team, 2017). Response variables were analyzed with different distribution families and link functions based on theoretical sampling distributions of the data, and model fits were validated with plots of model residuals, and were checked for collinearity.

306

We used a binomial distribution family and logit link function to analyze differences in discrimination performance and number of aborted trials, since these were binary data. We used an inverse Gaussian distribution family with an identity link function to analyze trial time data (Baayen and Milin, 2010). Log-normal linear models (Gaussian family with an identity function) were used to analyze log-transformed received call level, duration, peak frequency, and frequency centroid. Peak frequency and frequency centroid measure similar aspects of vocalization frequency and are both used in the literature (Goerlitz et al., 2008; Holderied et al., 2005; Lattenkamp et al., 2018; Lazure and Fenton, 2011). We therefore included both metrics in our analyses for comparability, but report only peak frequency in the main text because it is the most commonly used metric, and present frequency centroid data in the supplementary information (Table S6).

318

We initially checked that discrimination performance did not change between the first and last (pre- and post-treatment) silent conditions with a logistic regression including noise treatment (as factors) and ripple height. Since the performance across the two silent noise conditions did not change for any of the bats (see Results), we pooled these data for analysis, and thus present both 'silent' conditions together.

For all models we used noise treatment, ripple height, and their interaction as explanatory variables, while the number of days that bats were in our experiment was included as a covariate, all fitted as fixed effects. The number of days an animal is in an experiment may influence 
329

330

331

332

333

334

335

336

337

338

339

340

341

342

343

344

345

346

347

348

349

350

351

352

353

354

355

356

357

358

359

give up on difficult tasks. We thus included this term as a covariate in our model to control for it (and show its effect in the model output tables), but focused our main analysis and interpretation on more pertinent variables.

We fitted individual models for each bat, instead of single models for every response variable, with bats as random effects terms (e.g. Gomes et al., 2017), for two reasons. Firstly, it has been suggested that random effects terms should have a minimum of five groups; otherwise estimates of variance become imprecise (Harrison et al., 2018). As we only had four bats that completed the experiment, we were unable to fulfill this requirement. Secondly, and more importantly, fitting models to each individual bat allowed us to understand the nuanced differences between them, which an all-bats-combined model would not achieve. Since we fitted four models per response variable (one for each bat), we used conservative Bonferroni corrections to correct $p$ values for these multiple comparisons by multiplying $p$ values by four. All differences reported in results due to noise treatments are model estimates, and not differences in raw data.

\section{Performance thresholds}

We used a binomial generalized linear model with a probit link (constrained between 0.5 and 1 with the link function 'mafc.probit' in the R package 'psyphy'; Knoblauch, 2007) to generate estimates of ripple height thresholds at which bats exceed correct responses at least $70 \%$ of the time. For each bat, 1000 simulated discrimination performance ( 0 or 1$)$ datasets were generated based on the above model estimates for each bat, at each ripple height, within each noise treatment. Then the lower 0.025 and upper 0.975 percentiles of those data gave us a $95 \%$ confidence interval band around our performance threshold.

\section{Results}

\section{Discrimination performance}

All four bats learned to discriminate the smooth disc from the rippled disc with the highest ripples $(32 \mathrm{~mm})$ in silence (88-100\% correct), and showed reduced discrimination performance with decreasing ripple height (Figure 3, orange line; logistic model $p<0.001$; Table 1). In silence, performance dropped below our $70 \%$ threshold criterion for ripple heights around 
360

361

362

363

364

365

366

367

368

369

370

371

372

373

374

375

376

377

378

379

380

381

382

383

384

385 386

387

388

389

390

$7.9 \mathrm{~mm}$ (mean; range $=5.4-11.4 \mathrm{~mm}$; Figure 3; Table 2), matching the mean threshold found by (Baier et al., 2019) of $8.0 \mathrm{~mm}$ (range: $3.7-12.3 \mathrm{~mm}$ ). As the performance in silence did not change for any bat between the first and last silence condition (Bat A: $p=0.21, \mathrm{~B}: p=0.89, \mathrm{C}: p$ $=0.81, \mathrm{D}: p=0.39$ ), demonstrating a lack of order (e.g. learning) effects, we pooled all silence trials. Noise treatments did not change the discrimination performance of bats A and B (hereafter 'coping' bats; Table 1), and the 95\% confidence intervals of their thresholds in noise overlapped with those in silence. In contrast, discrimination performance decreased for bats $\mathrm{C}$ and D (hereafter 'non-coping' bats) both under smooth-overlapping noise $(\mathrm{z}=-3.2, p=0.008 ; \mathrm{z}=-3.2$, $p=0.004)$ and sparse-overlapping noise $(\mathrm{z}=-3.7, p<0.001 ; \mathrm{z}=-3.1, p=0.008$; indicated as blue and purple lines in Figure 3 respectively). The same is true for the smooth non-overlapping noise for bat $\mathrm{C}(\mathrm{z}=-2.5, p=0.047$, green line $)$, yet not for bat $\mathrm{D}(\mathrm{z}=1.8, p=0.24)$.

\section{Trial duration}

The time to complete trials differed between some noise treatments for some bats (Figure 4).

Both bats $\mathrm{A}$ and $\mathrm{D}$ made faster decisions during smooth-overlapping noise compared to silence (model estimated trial durations of bat $\mathrm{A}$ and $\mathrm{D}$ under noise and silence, respectively: $28.8 \mathrm{~s}$ vs. $30.0 \mathrm{~s}(\mathrm{~A})$ and $10.3 \mathrm{~s}$ vs. $14.8 \mathrm{~s}(\mathrm{D}) ; \mathrm{z}=-3.4, p=0.004$ (A); $\mathrm{z}=-4, p<0.001$ (D); Table S1). However, bat $\mathrm{C}$ took longer to complete trials during sparse-overlapping noise (48.6 s vs. $18.5 \mathrm{~s}$; $\mathrm{z}=4.8, p<0.001$ ), while noise treatments did not affect the trial time of bat B (Table S1).

\section{Aborted Trials}

The bats aborted 297 trials of 4,257 total trials (7 \%; bats A: 54; B: 92; C: 101; D: 50).

Compared to silence, both bats B and C significantly aborted more trials under both smooth nonoverlapping $(\mathrm{z}=4.0, p<0.001 ; \mathrm{z}=3.0, p=0.01)$ and smooth-overlapping noise $(\mathrm{z}=3.9, p<$ $0.001 ; \mathrm{z}=4.2, p<0.001)$. In addition, bat $\mathrm{B}$ and also bat $\mathrm{D}$ aborted more trials under sparseoverlapping noise compared to silence $(\mathrm{z}=5.2, p<0.001 ; \mathrm{z}=3.8, p<0.001$; Figure 5; Table S2).

\section{Echolocation call parameters}

We recorded at least one call for most (87.6\%) of the 3960 total trials, and at least ten calls for more than half of the trials $(57.2 \%)$, and used the mean call parameters of each trial in the 
391

392

393

394

395

396

397

398

399

400

401

402

403

404

405

406

407

408

409

410

411

412

413

414

415

416

417

418

419

420

421

analysis. Mean call duration ranged from $0.38 \mathrm{~ms}$ (bat A) to $0.47 \mathrm{~ms}$ (bat C), matching previous results in confined space (Goerlitz et al., 2008; Luo et al., 2015b). All bats increased call duration under smooth-overlapping noise. Coping bats (A and B) increased call duration by an estimated $0.07 \mathrm{~ms}$, while non-coping bats $(\mathrm{C}$ and $\mathrm{D})$ only increased call duration by $0.05 \mathrm{~ms}$ and 0.04 ms (bat A: $\mathrm{t}=20.4, p<0.001 ; \mathrm{B}: \mathrm{t}=9.1, p<0.001 ; \mathrm{C}: \mathrm{t}=7.9, p<0.001 ; \mathrm{D}: \mathrm{t}=6.0, p<$ 0.001; Table S3). Similarly, coping bats increased call duration in sparse-overlapping noise (increase of $0.06 \mathrm{~ms}$ and $0.07 \mathrm{~ms}, \mathrm{~A}$ and $\mathrm{B}$ respectively), while non-coping bats $\operatorname{did}$ not $(\mathrm{A}: \mathrm{t}=$ 15.1, $p<0.001 ; \mathrm{B}: \mathrm{t}=9.3, p<0.001 ; \mathrm{C}: \mathrm{t}=1.2, p=0.22 ; \mathrm{D}: \mathrm{t}=1.8, p=0.07$; Figure 6). Oddly, bats $\mathrm{B}$ and $\mathrm{C}$ decreased call duration by $0.03 \mathrm{~ms}$ and $0.02 \mathrm{~ms}$ in non-overlapping noise relative to silence $(\mathrm{B}: \mathrm{t}=-4.1, p<0.001 ; \mathrm{C}: \mathrm{t}=-3.8, p<0.001)$.

Relative to silence, all bats increased their call sound pressure level in both overlapping noise treatments by about 10-13 dB (smooth overlapping noise: bats A: $11.8 \mathrm{~dB}$; B: $9.7 \mathrm{~dB}$; C: $9.6 \mathrm{~dB}$; D: $13.3 \mathrm{~dB}(\mathrm{t}=46.1 ; \mathrm{t}=31.8 ; \mathrm{t}=33.0 ; \mathrm{t}=25.8)$; sparse overlapping noise: $\mathrm{A}: 12.6 \mathrm{~dB} ; \mathrm{B}: 8.7$ $\mathrm{dB} ; \mathrm{C}: 10.5 \mathrm{~dB} ; \mathrm{D}: 13.3 \mathrm{~dB} ;(\mathrm{t}=47.4 ; \mathrm{t}=29.8 ; \mathrm{t}=37.6 ; \mathrm{t}=19.8)$, all $p<0.001$; Figure 7; Table S4). Additionally, bat A also increased call level during the smooth non-overlapping noise, though by a much lower magnitude of only $1.5 \mathrm{~dB}(\mathrm{t}=4.7, p<0.001)$.

The mean peak frequency was $69.8 \mathrm{kHz}$ (bats A: $71.8 \mathrm{kHz}$; B: $69.9 \mathrm{kHz}$; C: $69.6 \mathrm{kHz}$; D: 67 $\mathrm{kHz}$ ). Of all 12 comparisons, only three showed significant, yet small changes of call frequency with no clear pattern: Bat A increased peak frequency in smooth non-overlapping noise by $1.2 \mathrm{kHz}$, and decreased peak frequency in smooth overlapping noise by $1.4 \mathrm{kHz}(\mathrm{t}=3.8, p<$ $0.001 ; \mathrm{t}=-4.5, p<0.001)$. Bat $\mathrm{C}$ increased peak frequency by $2.1 \mathrm{kHz}$ only in sparse overlapping noise $(\mathrm{t}=5.5, p<0.001$; Figure 8). Bats B and D never changed their peak frequency (Table S5).

\section{Discussion}

We tested the ability of four bats to discriminate increasingly rippled surface structures from a flat surface under silence and three different noise types. By comparing the bats' discrimination performance, behavior, and echolocation parameters, we address the perceptual mechanism of 
422 noise disturbance, and how bats may be able to cope with noise disturbance. The individual bats

423 in our experiments responded to noise in varying ways. Two bats (A and B; "coping") were able

424 to cope with all three noise types, as their discrimination performance was not affected by noise.

425 In contrast, the other two bats (C and D; "non-coping") were not able to cope with the noise, yet

426 in different ways. Bat $\mathrm{C}$ had decreased discrimination performance in all three noise types, took

427 longer in sparse-overlapping noise, and aborted more trials in smooth-overlapping and smooth

428 non-overlapping noise. Bat D had strongly reduced discrimination performance in both smooth

429 and sparse overlapping noise types (but not in non-overlapping noise), made faster decisions in

430 smooth-overlapping treatments, and aborted more trials only in sparse-overlapping noise. Of the

431 changes in echolocation call parameters, the increase in call level was the most prominent one,

432 and shown by both coping and non-coping bats in response to both overlapping noise types.

433 Changes in call frequency were much smaller and without a clear pattern, while call duration

434 increased slightly more for the coping than the non-coping bats. Based on our predictions both

435 perceptual mechanisms tested, masking and distraction, appeared to contribute to the bats'

436 performance. In the following, we will discuss all measured parameters in relation to our

437 predictions about the perceptual mechanisms of noise disturbance.

438

439

Discrimination performance (masking vs distraction)

440 We analyzed the ripple discrimination performance to address the perceptual mechanisms of

441 masking and distraction. Masking should only reduce the performance in overlapping noise, and

442 more so for smooth than sparse overlapping noise. In contrast, distraction should reduce the

443 performance in all noise types, and most so for sparse overlapping noise. Overall, our results do

444 not match those predictions: the coping bats (A and B) showed no decreased performance in any

445 of the noise treatments, excluding masking and distraction. Bat $\mathrm{C}$ seemed to suffer from

446 distraction, as its discrimination performance was affected by all noise types. In contrast to our

447 prediction, however, sparse overlapping noise did not reduce performance more than the other

448 noises. Lastly, bat D seemed to suffer from masking, as, in line with our prediction, its

449 discrimination performance was only reduced in both overlapping noise types - yet again

450 without difference between the smooth and sparse noise (in contrast to our prediction). The

451 sparse noise had temporal gaps with a mean duration of $0.3 \mathrm{~ms}$ (range: $0-0.6 \mathrm{~ms}$ ), which is

452 slightly shorter than the average $P$. discolor call here $(0.43 \mathrm{~ms}$ in silence). Although the detection 
453 performance of the gleaning bat Megaderma lyra for rustling sounds improved at around this gap

454 duration (Hübner and Wiegrebe, 2003), it is possible that the temporal gaps in the sparse noise

455 were not sufficiently long to provide sufficient release from masking for echo detection in our

456 species $P$. discolor, despite similarly short call durations of $<1 \mathrm{~ms}$ in confined spaces (Goerlitz

457 et al., 2008; Schuchmann et al., 2006). Therefore, our prediction that sparse-overlapping noise

458 would allow bats to listen in between the gaps of the noise may be false, and further tests with

459 larger gap widths are required. It is also possible that any release from masking that bats had

460 gained might have been opposed by an additional distracting effect of the sparse-overlapping

461 noise opposes, but this seems less likely than the lack of release from masking.

462

463 Trial duration (masking vs distraction)

464 To further differentiate between masking and distraction as perceptual mechanisms, we also

465 analyzed trial duration as a proxy for task difficulty. Only bat $\mathrm{C}$ showed a change in line with our

466 predictions, namely a 26\% increase in trial duration in sparse overlapping noise, indicative of

467 stronger distraction by this temporally fluctuating noise. This matches our previous interpretation

468 of this bat's discrimination performance, suggesting that this bat was mostly affected by

469 distraction, which should be strongest for the sparse noise. In contrast, the trial durations in

470 smooth-overlapping noise of both the coping bat A and the non-coping bat D was even shorter

471 than in silence, by 13 and $18 \%$, respectively. In the coping bat A, this faster decision making did

472 not reduce the discrimination performance, thus rather indicating reduced task difficulty due to

473 the smooth overlapping noise, which however seems unlikely. In the non-coping bat D, the

474 shorter trial duration might indicate the bat has stopped attempting to complete the task

475 accurately, due to the increased task difficulty by the smooth overlapping noise. This matches

476 our previous interpretation that this bat was affected by masking. However, it is unclear why this

477 bat had equally reduced discrimination performance in sparse overlapping noise, but trial

478 duration was not affected. In summary, trial duration partially supports distraction and masking

479 as perceptual mechanisms of noise disturbance for bats C and D, respectively, but this evidence 480 is not conclusive.

481

482 Echolocation call characteristics (masking) 
483 Several bat species change echolocation call parameters in response to noise (Bunkley et al., 484 2015; Hage et al., 2013; Luo et al., 2017; Tressler and Smotherman, 2009), which is a potential 485 mechanism to mitigate masking effects of noise (Brumm, 2013). Thus, we next discuss whether 486 the differences in coping ability (discrimination performance) can be explained by changes in 487 echolocation call parameters. The most prominent change was an increase in call level by around 488 10-13 dB, shown by all four bats (coping and non-coping) in both overlapping noise types 489 (smooth and sparse). This Lombard effect, the increase of vocalization amplitude in response to 490 noise, is found in many animals from birds to humans (Brumm and Zollinger, 2011). Our

491

492

493

494

495

496

497

498

499

500

501

502

503

504

505

506

507

508

509

510

511

512

513 species, Phyllostomus discolor, also exhibits an increasing Lombard effect with increasing noise level, amounting to on average $+4 \mathrm{~dB}$ for overlapping $(40-90 \mathrm{kHz})$ noise with a level of $52 \mathrm{~dB}$ SPL (Luo et al., 2015b). Here, we show that the Lombard effect increases even further up to 10$13 \mathrm{~dB}$ when noise levels are higher (70 dB SPL). This increase in call level is likely a direct response to masking (c.f. Fig 3 Brumm and Todt, 2002), as only one of the bats (bat A) increased call amplitude in non-masking noise, and this effect was an order of magnitude smaller $(+1.5 \mathrm{~dB}$, c.f. Luo et al., 2015b). Interestingly, however, although the reaction in call level was equal across all four bats, only two bats (A and B) were able to cope with masking overlapping noise in the discrimination task, while the other two bats $(\mathrm{C}$ and $\mathrm{D})$ showed strongly reduced discrimination performance. If we assume that the increased call amplitude provides equal release from masking for all four bats, another perceptual mechanism instead of masking must be responsible for the reduced discrimination performance of the non-coping bats.

In addition to increasing call level, increased call duration improves signal detection in noise because the mammalian ear integrates sound intensity over time (Heil and Neubauer, 2003). Indeed, bats increased call duration under noise both in laboratory (Luo et al., 2015b) and field environments (Bunkley et al., 2015). Here, our bats also increased call duration, and did so only in overlapping noise types, suggesting that this was a direct response to masking. We found some differences between coping and non-coping bats. While the coping bats increased call duration by $14-16 \%$ in both overlapping noise types (smooth and sparse), the non-coping bats increased their call duration only in the smooth overlapping noise, and only by $9 \%$. At first view, these patterns are consistent that coping bats mitigate noise masking by increasing call duration, while non-coping bats fail to do so. However, the rather small increase in call duration found 
514 here improves signal detectability by only about $1 \mathrm{~dB}$ (assuming a gain of $6 \mathrm{~dB}$ per doubling of

515 call duration; Luo et al., 2015b). This is much less than the direct increase in call level (10-13

$516 \mathrm{~dB}$ ) shown by both coping and non-coping bats, making it unlikely the slight differences in call

517 duration change can explain the differences in discrimination ability.

\section{8}

Shifting call frequency away from the frequency of a masker is another perceptual mechanism to improve signal detection by reducing spectral overlap, shown by bats when foraging in crowded situations (Bates et al., 2008; Gillam et al., 2007; Ratcliffe et al., 2004) or near loud ultrasonic insect choruses (Gillam and McCracken, 2007). In lower-frequency (5-35 kHz) non-overlapping noise, bat A indeed showed frequency changes consistent with avoiding spectral overlap by increasing its call peak frequency by $1.2 \mathrm{kHz}$. In contrast, the decrease of its peak frequency around $70 \mathrm{kHz}$ by $1.4 \mathrm{kHz}$ in the higher frequency $(40-90 \mathrm{kHz})$ smooth-overlapping noise is unlikely to improve signal detectability; and correspondingly this bat did not change its peak frequency in the other overlapping noise type (sparse). Bat $\mathrm{C}$ increased peak frequency in sparseoverlapping noise only; and the bats B and D showed no response. It is unlikely that such small $(\leq 2 \mathrm{kHz})$ changes in frequency have large effects on call detectability in noise, and thus do not seem insightful for making predictions on the ability of bats to cope with noise.

\section{Aborted trials}

533 Lastly, the bats avoided the noise types differently. While the coping bat A did not abort more 534 trials under any noise type compared to silence, the other coping bat B aborted more trials in all three noise types $(6.0,4.9$, and 11.8 times more in smooth non-overlapping, smooth-overlapping, and sparse-overlapping noise, respectively). This pattern is suggestive of the noise being interpreted as novel or dangerous, perhaps causing fear (i.e. misleading), since the noise type did not affect the discrimination performance and trial duration in this bat (which we would expect if the bat was masked or distracted). The two non-coping bats showed opposite patterns in the number of aborted trials. Bat $\mathrm{C}$ aborted more trials in both smooth noise types $(2.4$ and 5.9 times more in smooth non-overlapping and smooth overlapping noise, respectively), but not in sparse overlapping noise. The response of bat $\mathrm{C}$ might indicate that smooth noise types might be more

543 misleading or fear-inducing, as it cannot be linked to masking or distraction. In contrast, bat D 544 aborted more trials in the sparse overlapping noise only (3.9 times more), but not in the two 
545 smooth noise types. It is possible here that the sparse overlapping noise was more distracting

546 than the smooth overlapping noise, causing more trials to be aborted (somehow without affecting

547 discrimination performance).

548

\section{Conclusion}

550 Understanding how echolocating bats deal with noise pollution will be important for their

551 conservation. Additionally, such a mechanistic understanding may also inform the design of

552 noise-based deterrence systems to protect bats from being killed by wind turbines (Arnett et al.,

553 2013). Recent studies have shown that echolocating bats avoid noise in the field and lab, when it

554 is possible (Bunkley et al., 2015; Luo et al., 2015a; Schaub et al., 2008), but as noise sources

555 expand and foraging habitat shrinks, avoidance will become more difficult. Here, when

556 avoidance is impossible, we show that the effects of noise and the underlying perceptual

557 mechanism of disturbance differ at the individual level. It is likely that masking affected all bats,

558 as all of them strongly increased their call levels. However, only two of four bats were able to

559 maintain discrimination performance in noise. Therefore, other perceptual mechanisms, in

560 addition to masking, likely affect signal perception by bats in noise, and probably to different

561 extents for each individual.

562

563 By grouping all individuals of one species, we may miss important differences in how

564 individuals deal with noise (reviewed in Harding et al., 2019). By ignoring variation across

565 individuals, we may be missing the potential for rapid evolution to occur in response to

566 anthropogenic changes (Sih et al., 2011). Noise (or other sensory pollutants) can filter

567 individuals over time by selecting for individuals that can cope with noise. Understanding the

568 variation in the ability to cope with noise is paramount to predicting which species may adapt

569 well to encroaching urbanization, and which will not. It is possible that this variation is

570 maintained in natural systems by individual microhabitat selection, because although natural

571 noise is ubiquitous in nature, it is spatially and temporally heterogeneous across the landscape.

572

573

574 Acknowledgements 
575 We would like to thank A. Leonie Baier for help building the experimental setup and training the 576 bats, Karin 'Reni' Heckel for assistance with animal care, Yossi Yovel and Stefan Greif for help 577 measuring light levels, and Jesse R. Barber, Henrik Brumm and two anonymous reviewers for 578 comments on earlier versions of the manuscript (see Gomes and Goerlitz, 2020a). We thank the 579 Max Planck Institute for Ornithology, Seewiesen, for excellent infrastructure and support, and 580 the Fulbright Program, the National Science Foundation (GRFP ID 2018268606 to DGEG) and 581 the Deutsche Forschungsgemeinschaft (DFG, German Research Foundation, Emmy Noether 582 grant 241711556 to HRG) for funding. All data and code are available on Zenodo (DOI:

583 10.5281/zenodo.3928601; Gomes and Goerlitz, 2020b). 584 
585

586

587

588

589

590

591

592

593

594

595

596

597

598

599

600

601

602

603

604

605

606

607

608

609

610

611

612

613

614

615

616

617

\section{References}

Arnett, E. B., Hein, C. D., Schirmacher, M. R., Huso, M. M. and Szewczak, J. M. (2013). Evaluating the effectiveness of an ultrasonic acoustic deterrent for reducing bat fatalities at wind turbines. PloS One $\mathbf{8}$.

Au, W. W. (2012). The sonar of dolphins. Springer Science \& Business Media.

Baayen, R. H. and Milin, P. (2010). Analyzing reaction times. Int. J. Psychol. Res. 3, 12-28.

Baier, A. L., Wiegrebe, L. and Goerlitz, H. R. (2019). Echo-Imaging Exploits an Environmental High-Pass Filter to Access Spatial Information with a Non-Spatial Sensor. IScience 14, 335-344.

Barber, J. R., Crooks, K. R. and Fristrup, K. M. (2010). The costs of chronic noise exposure for terrestrial organisms. Trends Ecol. Evol. 25, 180-189.

Bates, M. E., Stamper, S. A. and Simmons, J. A. (2008). Jamming avoidance response of big brown bats in target detection. J. Exp. Biol. 211, 106-113.

Bell, G. P. and Fenton, M. B. (1986). Visual acuity, sensitivity and binocularity in a gleaning insectivorous bat, Macrotus californicus (Chiroptera: Phyllostomidae). Anim. Behav. 34, 409-414.

Bondello, M. C. and Brattstrom, B. H. (1978). Ambient sound pressure levels in the California desert. Rep. Prep. Bur. Land Manag. Contract CA-060-CT7-2737.

Boyles, J. G., Cryan, P. M., McCracken, G. F. and Kunz, T. H. (2011). Economic importance of bats in agriculture. Science 332, 41-42.

Bruintjes, R. and Radford, A. N. (2013). Context-dependent impacts of anthropogenic noise on individual and social behaviour in a cooperatively breeding fish. Anim. Behav. 85, 13431349.

Brumm, H. (2013). Animal communication and noise. Springer.

Brumm, H. and Slabbekoorn, H. (2005). Acoustic communication in noise. Adv. Study Behav. 35, 151-209.

Brumm, H. and Todt, D. (2002). Noise-dependent song amplitude regulation in a territorial songbird. Anim. Behav. 63, 891-897.

Brumm, H. and Zollinger, S. A. (2011). The evolution of the Lombard effect: 100 years of psychoacoustic research. Behaviour 1173-1198.

Bunkley, J. P. and Barber, J. R. (2015). Noise reduces foraging efficiency in pallid bats (Antrozous pallidus). Ethology 121, 1116-1121. 
618

619

620

621

622

623

624

625

626

627

628

629

630

631

632

633

634

635

636

637

638

639

640

641

642

643

644

645

646

647

648

649

650

651

652

Bunkley, J. P., McClure, C. J., Kleist, N. J., Francis, C. D. and Barber, J. R. (2015). Anthropogenic noise alters bat activity levels and echolocation calls. Glob. Ecol. Conserv. 3, 62-71.

Buxton, R. T., McKenna, M. F., Mennitt, D., Fristrup, K., Crooks, K., Angeloni, L. and Wittemyer, G. (2017). Noise pollution is pervasive in US protected areas. Science 356, $531-533$.

Campo, J. L., Gil, M. G. and Davila, S. G. (2005). Effects of specific noise and music stimuli on stress and fear levels of laying hens of several breeds. Appl. Anim. Behav. Sci. 91, 7584.

Chan, A. A. Y.-H., Giraldo-Perez, P., Smith, S. and Blumstein, D. T. (2010). Anthropogenic noise affects risk assessment and attention: the distracted prey hypothesis. Biol. Lett. 6 , $458-461$.

Christy, J. H. (1995). Mimicry, mate choice, and the sensory trap hypothesis. Am. Nat. 146, $171-181$.

Cinto Mejia, E., McClure, C. J. and Barber, J. R. (2019). Large-scale manipulation of the acoustic environment can alter the abundance of breeding birds: Evidence from a phantom natural gas field. J. Appl. Ecol. 56, 2091-2101.

Clark, C. W., Ellison, W. T., Southall, B. L., Hatch, L., Van Parijs, S. M., Frankel, A. and Ponirakis, D. (2009). Acoustic masking in marine ecosystems: intuitions, analysis, and implication. Mar. Ecol. Prog. Ser. 395, 201-222.

Dominoni, D. M., Halfwerk, W., Baird, E., Buxton, R. T., Fernández-Juricic, E., Fristrup, K. M., McKenna, M. F., Mennitt, D. J., Perkin, E. K. and Seymoure, B. M. (2020). Why conservation biology can benefit from sensory ecology. Nat. Ecol. Evol. 1-10.

Eastcott, E., Kern, J. M., Morris-Drake, A. and Radford, A. N. (2020). Intrapopulation variation in the behavioral responses of dwarf mongooses to anthropogenic noise. Behav. Ecol.

Eklöf, J., Šuba, J., Petersons, G. and Rydell, J. (2014). Visual acuity and eye size in five European bat species in relation to foraging and migration strategies. Env. Exp Biol 12, $1-6$.

Esser, K.-H. and Daucher, A. (1996). Hearing in the FM-bat Phyllostomus discolor: a behavioral audiogram. J. Comp. Physiol. A 178, 779-785.

Fay, R. R. and Wilber, L. A. (1989). Hearing in vertebrates: a psychophysics databook. Acoustical Society of America.

Fenton, M. B. and Bell, G. P. (1981). Recognition of species of insectivorous bats by their echolocation calls. J. Mammal. 62, 233-243.

Peer) reviewing PDF | (2020:09:52735:1:0:NEW 13 Nov 2020) 
653

654

655

656

657

658

659

660

661

662

663

664

665

666

667

668

669

670

671

672

673

674

675

676

677

678

679

680

681

682

683

684

685

Francis, C. D., Ortega, C. P. and Cruz, A. (2011). Noise pollution filters bird communities based on vocal frequency. PLoS One 6, e27052.

Francis, C. D., Kleist, N. J., Ortega, C. P. and Cruz, A. (2012). Noise pollution alters ecological services: enhanced pollination and disrupted seed dispersal. Proc. R. Soc. B Biol. Sci. 279, 2727-2735.

Furnham, A. and Strbac, L. (2002). Music is as distracting as noise: the differential distraction of background music and noise on the cognitive test performance of introverts and extraverts. Ergonomics 45, 203-217.

Gellermann, L. W. (1933). Chance orders of alternating stimuli in visual discrimination experiments. J. Genet. Psychol. 42, 206-208.

Gillam, E. H. and McCracken, G. F. (2007). Variability in the echolocation of Tadarida brasiliensis: effects of geography and local acoustic environment. Anim. Behav. 74, 277 286.

Gillam, E. H., Ulanovsky, N. and McCracken, G. F. (2007). Rapid jamming avoidance in biosonar. Proc. R. Soc. B Biol. Sci. 274, 651-660.

Glass, D. C. and Singer, J. E. (1972). Behavioral Aftereffects of Unpredictable and Uncontrollable Aversive Events: Although subjects were able to adapt to loud noise and other stressors in laboratory experiments, they clearly demonstrated adverse aftereffects. Am. Sci. 60, 457-465.

Goerlitz, H. R., Hübner, M. and Wiegrebe, L. (2008). Comparing passive and active hearing: spectral analysis of transient sounds in bats. J. Exp. Biol. 211, 1850-1858.

Gomes, D. G. and Goerlitz, H. R. (2020a). Individual differences show that only some bats can cope with noise-induced masking and distraction. bioRxiv.

Gomes, D. G. E. and Goerlitz, H. R. (2020b). Data and code for: Individual differences show that only some bats can cope with noise-induced masking and distraction.

Gomes, D. G., Halfwerk, W., Taylor, R. C., Ryan, M. J. and Page, R. A. (2017). Multimodal weighting differences by bats and their prey: probing natural selection pressures on sexually selected traits. Anim. Behav. 134, 99-102.

Gomes, D. G., Page, R. A., Geipel, I., Taylor, R. C., Ryan, M. J. and Halfwerk, W. (2016). Bats perceptually weight prey cues across sensory systems when hunting in noise. Science 353, 1277-1280.

Grunwald, J.-E., Schörnich, S. and Wiegrebe, L. (2004). Classification of natural textures in echolocation. Proc. Natl. Acad. Sci. 101, 5670-5674. 
686

687

688

689

690

691

692

693

694

695

696

697

698

699

700

701

702

703

704

705

706

707

708

709

710

711

712

713

714

715

716

717

718

Hage, S. R., Jiang, T., Berquist, S. W., Feng, J. and Metzner, W. (2013). Ambient noise induces independent shifts in call frequency and amplitude within the Lombard effect in echolocating bats. Proc. Natl. Acad. Sci. 110, 4063-4068.

Halfwerk, W., Holleman, L. J. and Slabbekoorn, H. (2011). Negative impact of traffic noise on avian reproductive success. J. Appl. Ecol. 48, 210-219.

Harding, H. R., Gordon, T. A., Eastcott, E., Simpson, S. D. and Radford, A. N. (2019). Causes and consequences of intraspecific variation in animal responses to anthropogenic noise. Behav. Ecol. 30, 1501-1511.

Harrison, X. A., Donaldson, L., Correa-Cano, M. E., Evans, J., Fisher, D. N., Goodwin, C. E., Robinson, B. S., Hodgson, D. J. and Inger, R. (2018). A brief introduction to mixed effects modelling and multi-model inference in ecology. PeerJ 6, e4794.

Hartmann, W. M. and Pumplin, J. (1988). Noise power fluctuations and the masking of sine signals. J. Acoust. Soc. Am. 83, 2277-2289.

Heil, P. and Neubauer, H. (2003). A unifying basis of auditory thresholds based on temporal summation. Proc. Natl. Acad. Sci. 100, 6151-6156.

Hoffmann, S., Baier, L., Borina, F., Schuller, G., Wiegrebe, L. and Firzlaff, U. (2008). Psychophysical and neurophysiological hearing thresholds in the bat Phyllostomus discolor. J. Comp. Physiol. A 194, 39-47.

Holderied, M. W., Korine, C., Fenton, M. B., Parsons, S., Robson, S. and Jones, G. (2005). Echolocation call intensity in the aerial hawking bat Eptesicus bottae (Vespertilionidae) studied using stereo videogrammetry. J. Exp. Biol. 208, 1321-1327.

Hübner, M. and Wiegrebe, L. (2003). The effect of temporal structure on rustling-sound detection in the gleaning bat, Megaderma lyra. J. Comp. Physiol. A 189, 337-346.

Kjellberg, A., Landström, U. L. F., Tesarz, M., Söderberg, L. and Akerlund, E. (1996). The effects of nonphysical noise characteristics, ongoing task and noise sensitivity on annoyance and distraction due to noise at work. J. Environ. Psychol. 16, 123-136.

Knoblauch, K. (2007). psyphy: Functions for analyzing psychophysical data in R. R Package Version 00-5 URL HttpCRAN R-Proj. Orgpackage Psyphy.

Kwiecinski, G. G. (2006). Phyllostomus discolor. Mamm. Species 2006, 1-11.

Lattenkamp, E. Z., Vernes, S. C. and Wiegrebe, L. (2018). Volitional control of social vocalisations and vocal usage learning in bats. J. Exp. Biol. 221,.

Lazure, L. and Fenton, M. B. (2011). High duty cycle echolocation and prey detection by bats. J. Exp. Biol. 214, 1131-1137. 
719

720

721

722

723

724

725

726

727

728

729

730

731

732

733

734

735

736

737

738

739

740

741

742

743

744

745

746

747

748

749

750

751

752

Luo, J., Siemers, B. M. and Koselj, K. (2015a). How anthropogenic noise affects foraging. Glob. Change Biol. 21, 3278-3289.

Luo, J., Goerlitz, H. R., Brumm, H. and Wiegrebe, L. (2015b). Linking the sender to the receiver: vocal adjustments by bats to maintain signal detection in noise. Sci. Rep. $\mathbf{5}$, 18556.

Luo, J., Lingner, A., Firzlaff, U. and Wiegrebe, L. (2017). The Lombard effect emerges early in young bats: Implications for the development of audio-vocal integration. J. Exp. Biol. 220, 1032-1037.

Luther, D. and Baptista, L. (2009). Urban noise and the cultural evolution of bird songs. Proc. R. Soc. B Biol. Sci. 277, 469-473.

Matthews, K. A., Scheier, M. F., Brunson, B. I. and Carducci, B. (1980). Attention, unpredictability, and reports of physical symptoms: Eliminating the benefits of predictability. J. Pers. Soc. Psychol. 38, 525.

Morris-Drake, A., Kern, J. M. and Radford, A. N. (2016). Cross-modal impacts of anthropogenic noise on information use. Curr. Biol. 26, R911-R912.

Naguib, M., van Oers, K., Braakhuis, A., Griffioen, M., de Goede, P. and Waas, J. R. (2013). Noise annoys: effects of noise on breeding great tits depend on personality but not on noise characteristics. Anim. Behav. 85, 949-956.

Nemeth, E. and Brumm, H. (2010). Birds and anthropogenic noise: are urban songs adaptive? Am. Nat. 176, 465-475.

Purser, J. and Radford, A. N. (2011). Acoustic noise induces attention shifts and reduces foraging performance in three-spined sticklebacks (Gasterosteus aculeatus). PLOS One 6 , e17478.

R Core Team (2017). $R$ : A language and environment for statistical computing. Vienna, Austria: R Foundation for Statistical Computing; 2016.

Rabin, L. A., McCowan, B., Hooper, S. L. and Owings, D. H. (2003). Anthropogenic noise and its effect on animal communication: an interface between comparative psychology and conservation biology. Int. J. Comp. Psychol. 16, 172-192.

Ratcliffe, J. M., Hofstede, H. M. ter, Avila-Flores, R., Fenton, M. B., McCracken, G. F., Biscardi, S., Blasko, J., Gillam, E., Orprecio, J. and Spanjer, G. (2004). Conspecifics influence call design in the Brazilian free-tailed bat, Tadarida brasiliensis. Can. J. Zool. 82, 966-971.

Schaub, A., Ostwald, J. and Siemers, B. M. (2008). Foraging bats avoid noise. J. Exp. Biol. 211, 3174-3180. 
753

754

755

756

757

758

759

760

761

762

763

764

765

766

767

768

769

770

771

772

773

774

775

776

777

778

779

780

781

782

783

784

785

786

787

Schuchmann, M., Hübner, M. and Wiegrebe, L. (2006). The absence of spatial echo suppression in the echolocating bats Megaderma lyra and Phyllostomus discolor. J. Exp. Biol. 209, 152-157.

Siemers, B. M. and Schaub, A. (2011). Hunting at the highway: traffic noise reduces foraging efficiency in acoustic predators. Proc. R. Soc. Lond. B Biol. Sci. 278, 1646-1652.

Sierro, J., Schloesing, E., Pavón, I. and Gil, D. (2017). European blackbirds exposed to aircraft noise advance their chorus, modify their song and spend more time singing. Front. Ecol. Evol. 5, 68 .

Sih, A., Ferrari, M. C. and Harris, D. J. (2011). Evolution and behavioural responses to human-induced rapid environmental change. Evol. Appl. 4, 367-387.

Simmons, A. M., Ertman, A., Hom, K. N. and Simmons, J. A. (2018). Big brown bats (Eptesicus fuscus) successfully navigate through clutter after exposure to intense bandlimited sound. Sci. Rep. 8, 1-13.

Simpson, S. D., Radford, A. N., Nedelec, S. L., Ferrari, M. C., Chivers, D. P., McCormick, M. I. and Meekan, M. G. (2016). Anthropogenic noise increases fish mortality by predation. Nat. Commun. 7, 10544.

Song, S., Chang, Y., Wang, D., Jiang, T., Feng, J. and Lin, A. (2020). Chronic traffic noise increases food intake and alters gene expression associated with metabolism and disease in bats. J. Appl. Ecol. 57, 1915-1925.

Standing, L., Lynn, D. and Moxness, K. (1990). Effects of noise upon introverts and extroverts. Bull. Psychon. Soc. 28, 138-140.

Tanner Jr, W. P. (1958). What is masking? J. Acoust. Soc. Am. 30, 919-921.

Tressler, J. and Smotherman, M. S. (2009). Context-dependent effects of noise on echolocation pulse characteristics in free-tailed bats. J. Comp. Physiol. A 195, 923-934.

Tyack, P. L., Zimmer, W. M., Moretti, D., Southall, B. L., Claridge, D. E., Durban, J. W., Clark, C. W., D’Amico, A., DiMarzio, N. and Jarvis, S. (2011). Beaked whales respond to simulated and actual navy sonar. PloS One 6, e17009.

Vélez, A. and Bee, M. A. (2011). Dip listening and the cocktail party problem in grey treefrogs: signal recognition in temporally fluctuating noise. Anim. Behav. 82, 1319-1327.

Voellmy, I. K., Purser, J., Flynn, D., Kennedy, P., Simpson, S. D. and Radford, A. N. (2014). Acoustic noise reduces foraging success in two sympatric fish species via different mechanisms. Anim. Behav. 89, 191-198.

West-Eberhard, M. J. (1984). Sexual selection, competitive communication and species specific signals in insects. In Insect Communication (Proceedings of the 12th Symposium of the Royal Entomological Society of London, p. Acedemic Press.

Peer) reviewing PDF | (2020:09:52735:1:0:NEW 13 Nov 2020) 
788 Wiley, R. H. (2013). Signal detection, noise, and the evolution of communication. In Animal communication and noise, pp. 7-30. Springer.

790

Zhou, Y., Radford, A. N. and Magrath, R. D. (2019). Why does noise reduce response to

791 alarm calls? Experimental assessment of masking, distraction and greater vigilance in wild birds. Funct. Ecol. 33, 1280-1289. 


\section{Figure 1}

Experimental setup and stimuli of the discrimination experiment.

A) Sketch of the experimental setup. The bat could freely move within the mushroom-shaped mesh-cage, allowing simultaneous perceptual access to both discs from multiple angles. Each trial started when the bat left from within the starting box and ended when the bat crossed an infrared light barrier next to each disc, objectively determining decision and duration of each trial. The bat received a food reward for approaching the flat reference disk. Noise treatments were presented via two speakers from similar directions as the returning disc echoes. Echolocation calls were recorded via microphones next to the light barriers. B) Cross-sections of the stimulus discs. The peak-to-peak height of the ripples is scaled to size and given on the right (but the shown distance between ripple peaks is shorter, i.e., higher spatial frequency, than in the experiment for better visualization). As ripple height gets smaller, the task to discriminate the rippled disk from the flat reference disc becomes more difficult, as indicated by the arrow. 


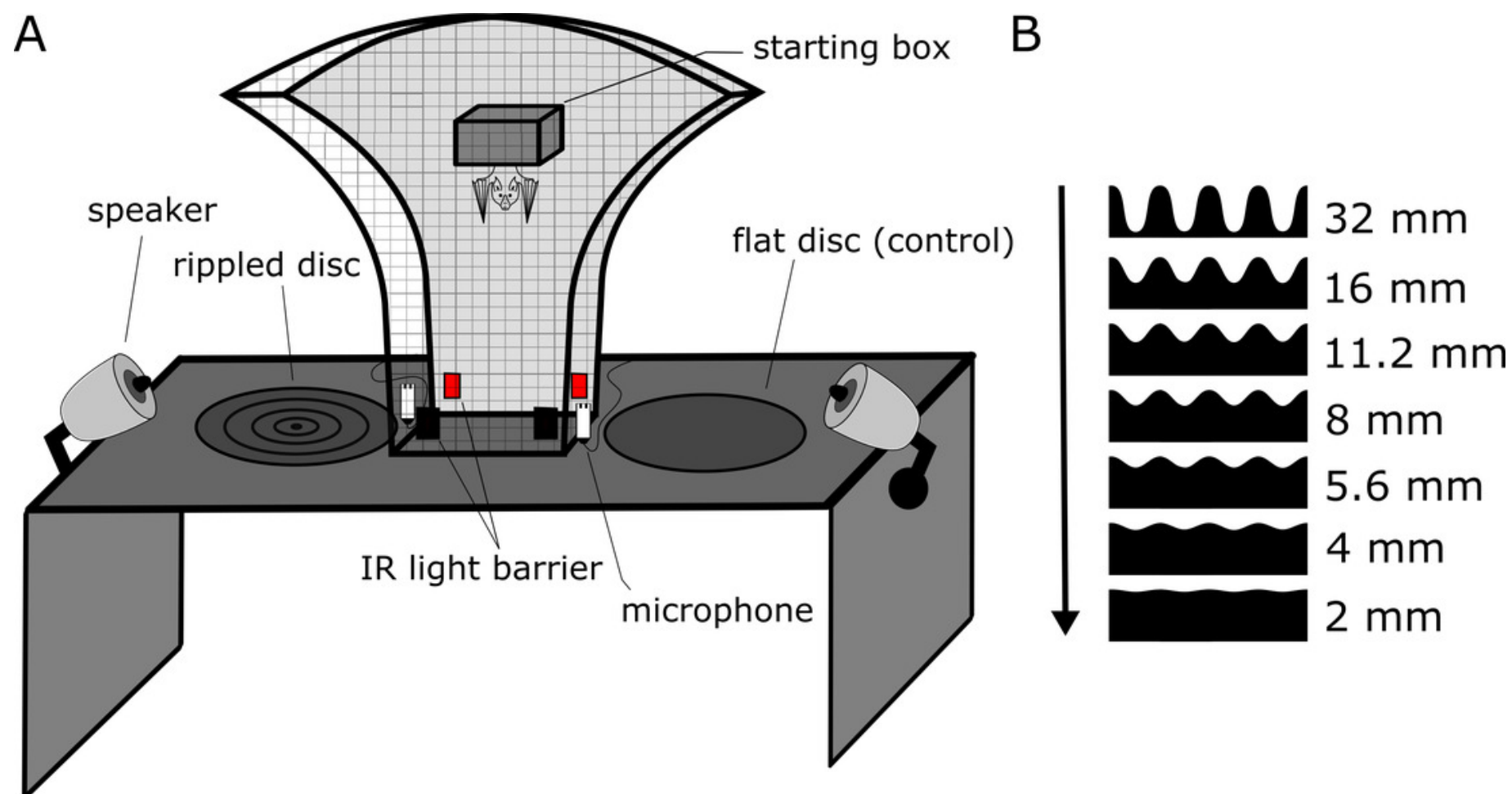




\section{Figure 2}

Experimental playback treatments.

Representative echolocation calls of Phyllostomus discolor in A) silence (control), B) smooth non-overlapping noise (5-35 kHz), C) smooth overlapping noise (40-90 kHz), and D) sparse overlapping noise (40-90 kHz with additional silent gaps of variable duration introduced between all adjacent noise samples; the duration of the silent gaps was drawn from a uniform distribution that ranged from 0 to $0.6 \mathrm{~ms}$ and had a mean value of $0.3 \mathrm{~ms}$ ). Echolocation call is based on Goerlitz et al. 2008. 
A

B
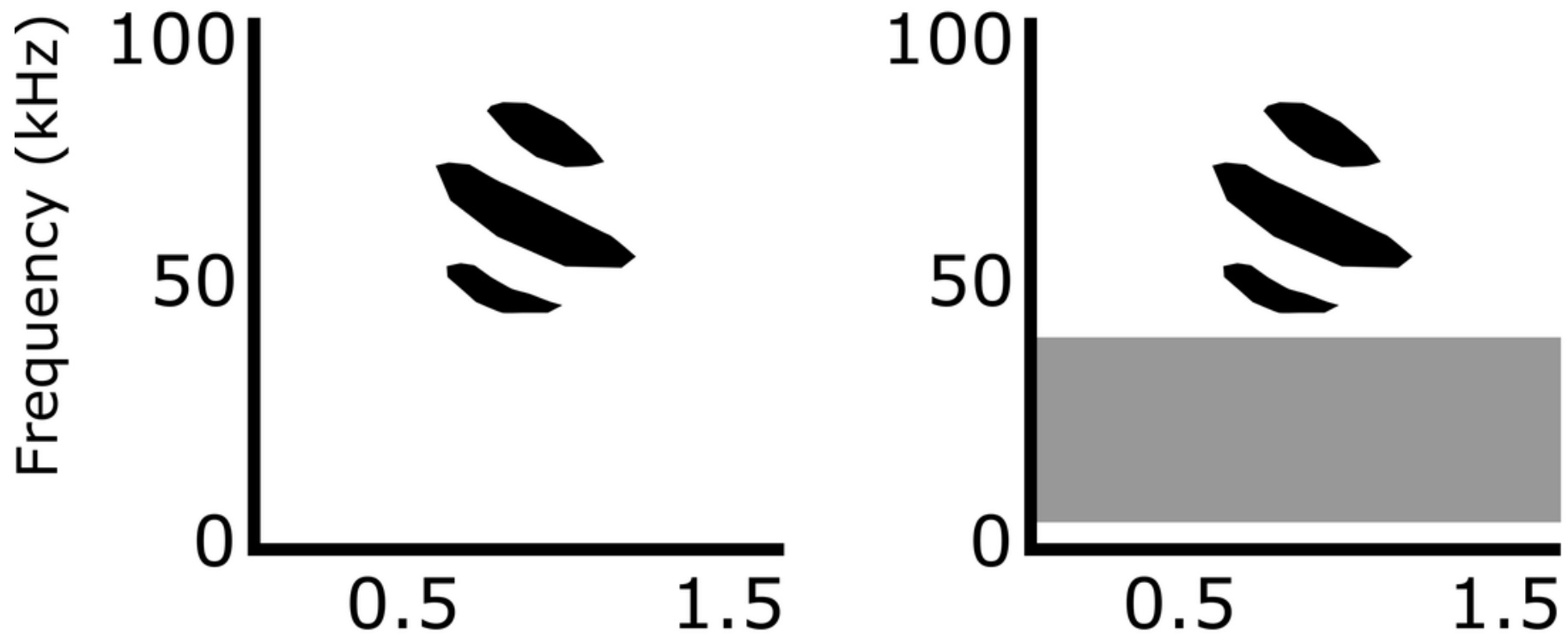

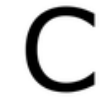

D

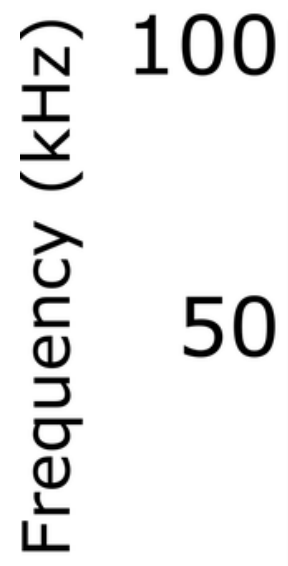

100
50

0

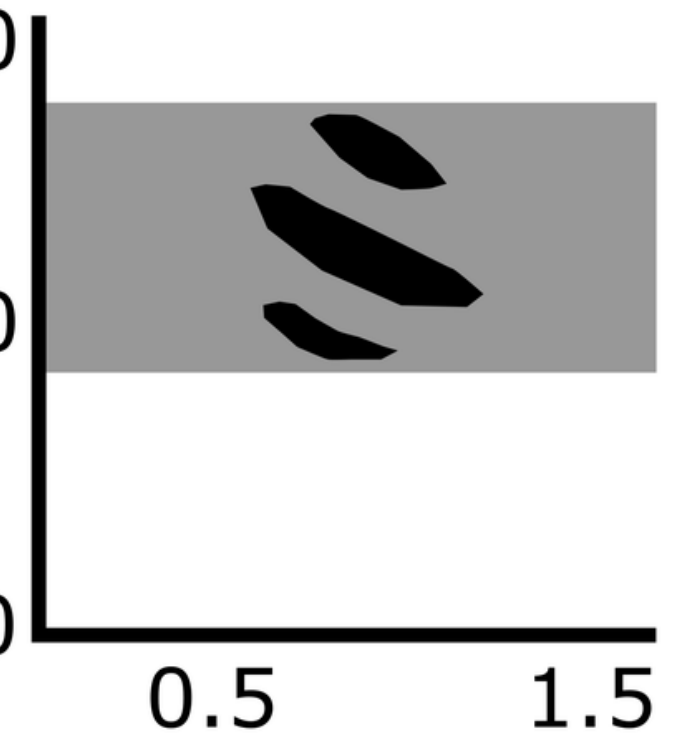

Time (ms)

Time (ms) 


\section{Figure 3}

Discrimination performance of four bats as a function of peak-to-peak disc ripple height during silent control and three noise treatments.

Shown are raw data (dots) and the fitted performance curve (colored lines; constrained probit function) for each noise treatment. The discrimination threshold is indicated by the vertical line, with its 95\% confidence interval. Discrimination performance of 'coping' bats ( $A$ and B) did not differ between silence and noise treatments. In contrast, discrimination performance of 'non-coping' bats ( $C$ and $D$ ) was reduced under both overlapping noise treatments (blue and purple lines), while discrimination performance of bat $\mathrm{C}$ was also reduced in nonoverlapping smooth noise (green line). Noise treatments have been abbreviated here (as compared to the text) to reduce visual clutter (smooth non-overlapping noise $=$ "Nonoverlap"; smooth-overlapping noise = "Smooth-overlap"; sparse-overlapping noise $=$ "Sparse-overlap"). Asterisks denote that the interaction between noise treatment and ripple height differs significantly $(p<0.05)$ from the silent controls (orange lines) in generalized linear models. 


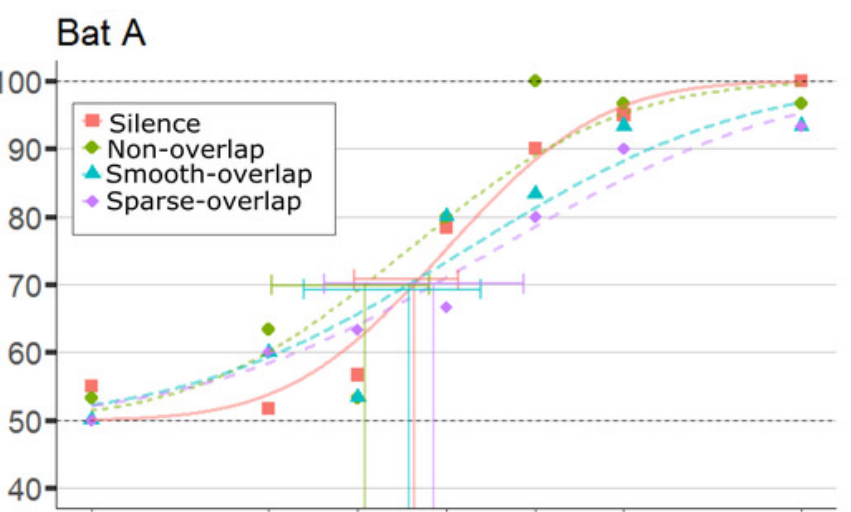

\section{Bat B}

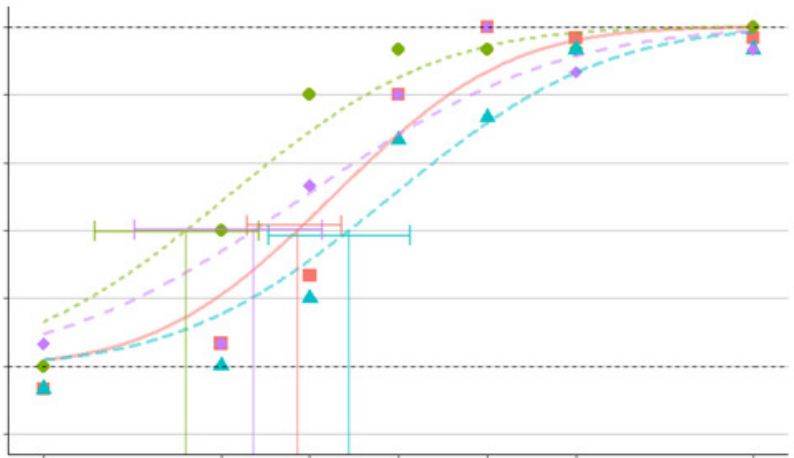

ฮे

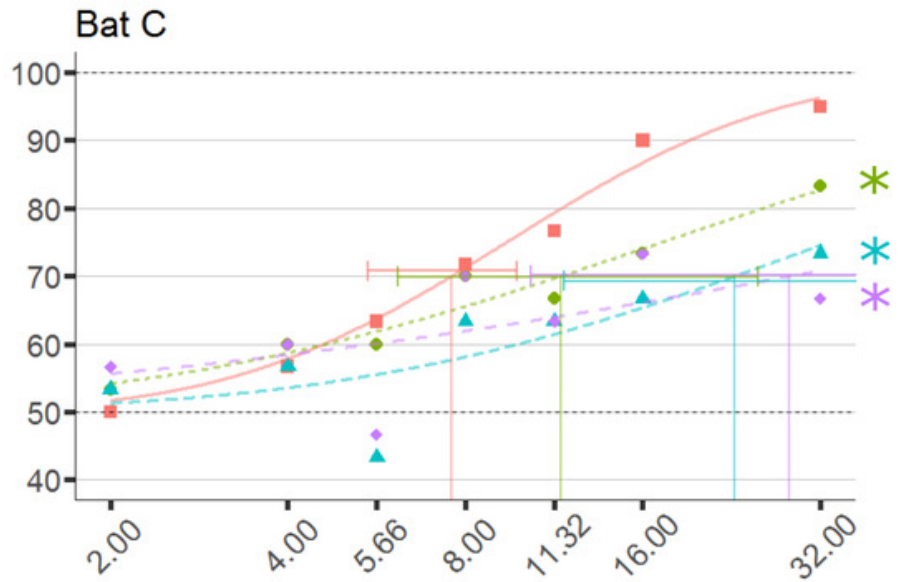

Bat D

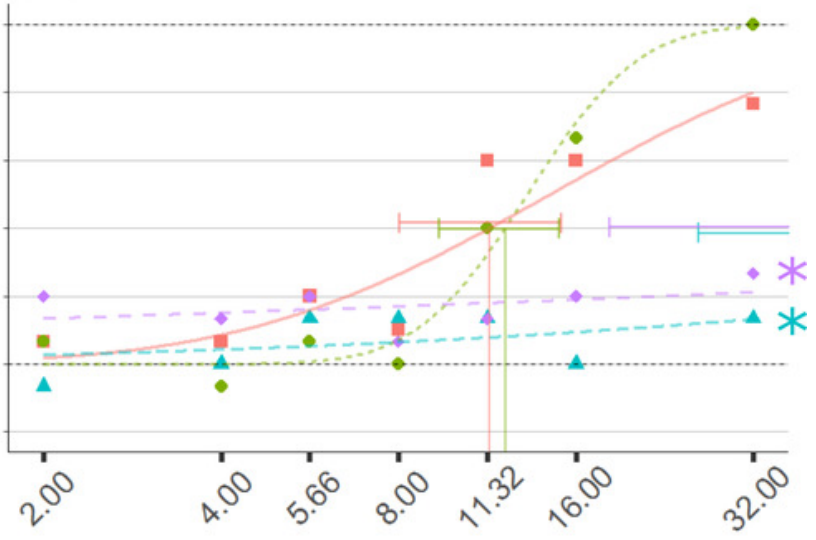

Ripple height (mm) 


\section{Figure 4}

Trial duration of the discrimination task by noise treatment.

Smooth-overlapping noise reduced trial duration of bats $A$ and $D$, and sparse-overlapping noise increased trial duration of bat C. Box plots show median (solid line) and first and third quartiles (edges of box); whiskers extend to the rest of the data minus outliers (more than 1.5 times the interquartile range), which are shown as points. Noise treatments have been abbreviated here (as compared to the text) to reduce visual clutter (smooth non-overlapping noise = "Non-overlap"; smooth-overlapping noise = "Smooth-overlap"; sparse-overlapping noise $=$ "Sparse-overlap"). Asterisks denote significant differences $(p<0.05)$ in trial duration relative to the silence control. 


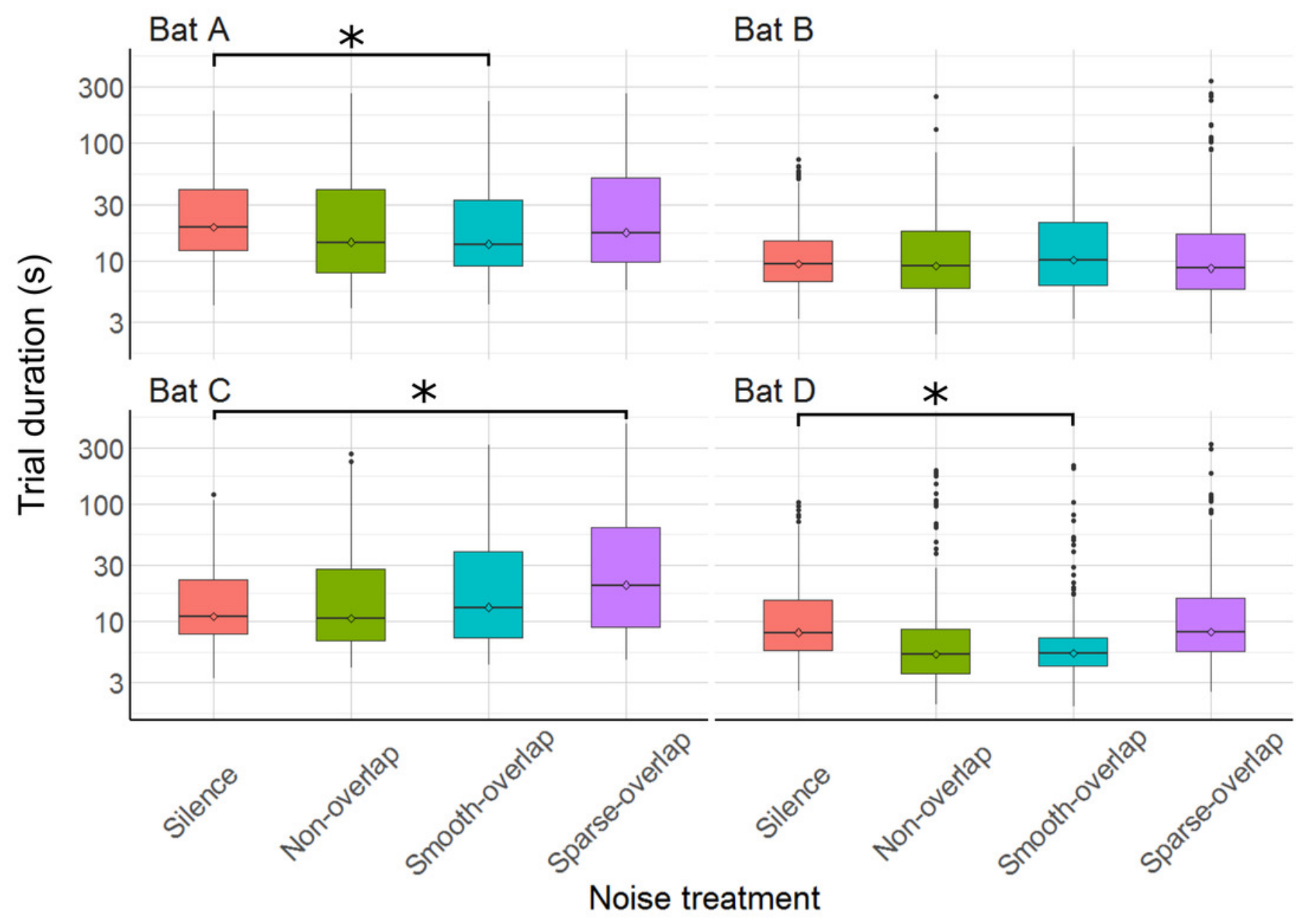




\section{Figure 5}

Percentage of aborted trials during the discrimination task by noise treatment.

Both bats $B$ and $C$ aborted significantly more trials for both non-overlapping and overlapping noise. Yet, bats $B$ and $D$ aborted significantly more trials for sparse-overlapping noise. Box plots show median (solid line) and first and third quartiles (edges of box); whiskers extend to the rest of the data minus outliers (more than 1.5 times the interquartile range), which are shown as points. Noise treatments have been abbreviated here (as compared to the text) to reduce visual clutter (smooth non-overlapping noise = "Non-overlap"; smooth-overlapping noise = "Smooth-overlap"; sparse-overlapping noise = "Sparse-overlap"). Asterisks denote significant differences $(p<0.05)$ in the percentage of aborted trials relative to the silence control. 


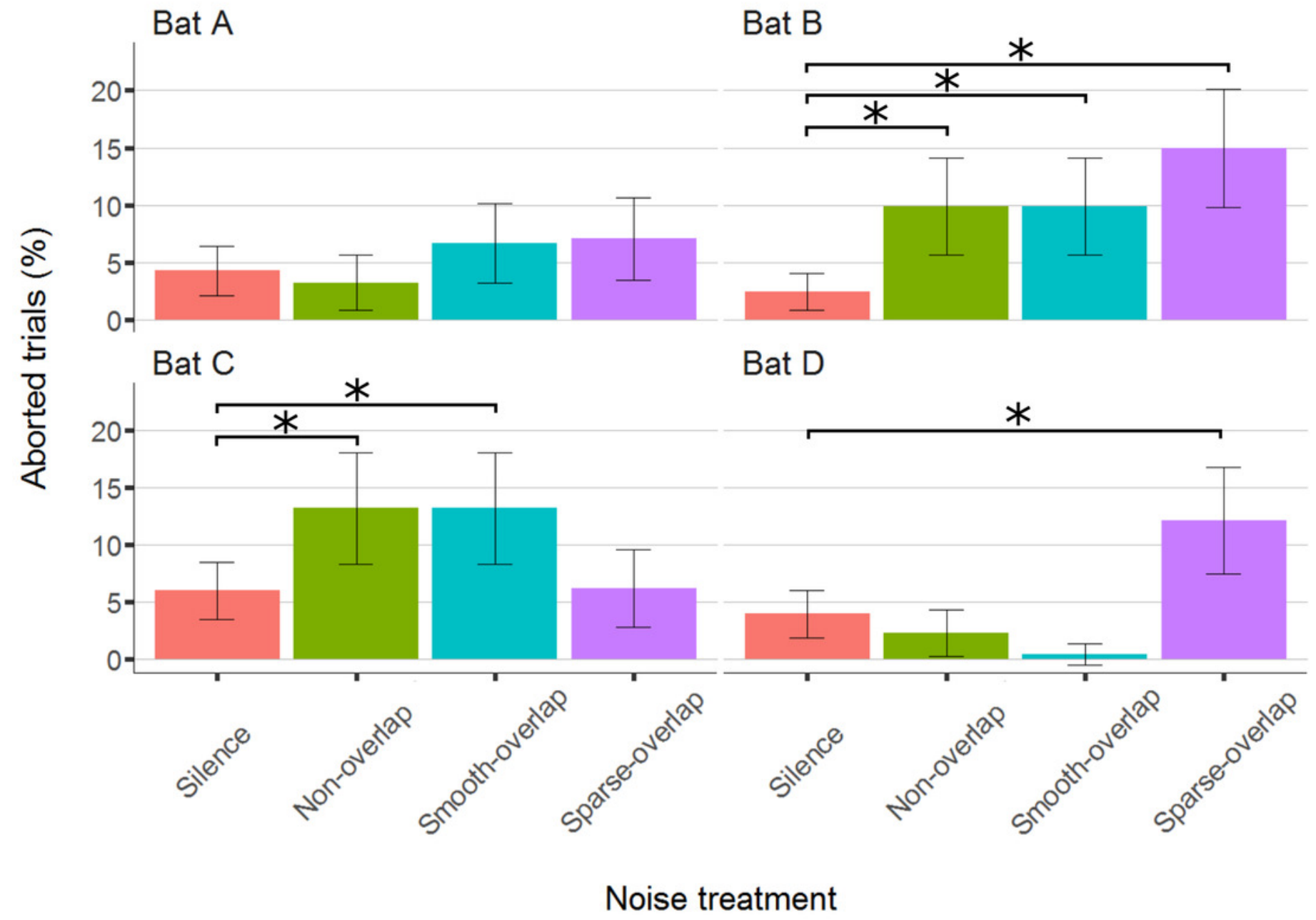




\section{Figure 6}

Duration of echolocation calls during the discrimination tasks by noise treatment.

All bats increased call duration under smooth-overlapping noise, and bats $A$ and $B$ also increased call duration under sparse-overlapping noise. Box plots show median (solid line) and first and third quartiles (edges of box); whiskers extend to the rest of the data minus outliers (more than 1.5 times the interquartile range), which are shown as points. Noise treatments have been abbreviated here (as compared to the text) to reduce visual clutter (smooth non-overlapping noise $=$ "Non-overlap"; smooth-overlapping noise $=$ "Smoothoverlap"; sparse-overlapping noise = "Sparse-overlap"). Asterisks indicate significant differences $(p<0.05)$ of call duration relative to the silence control. 


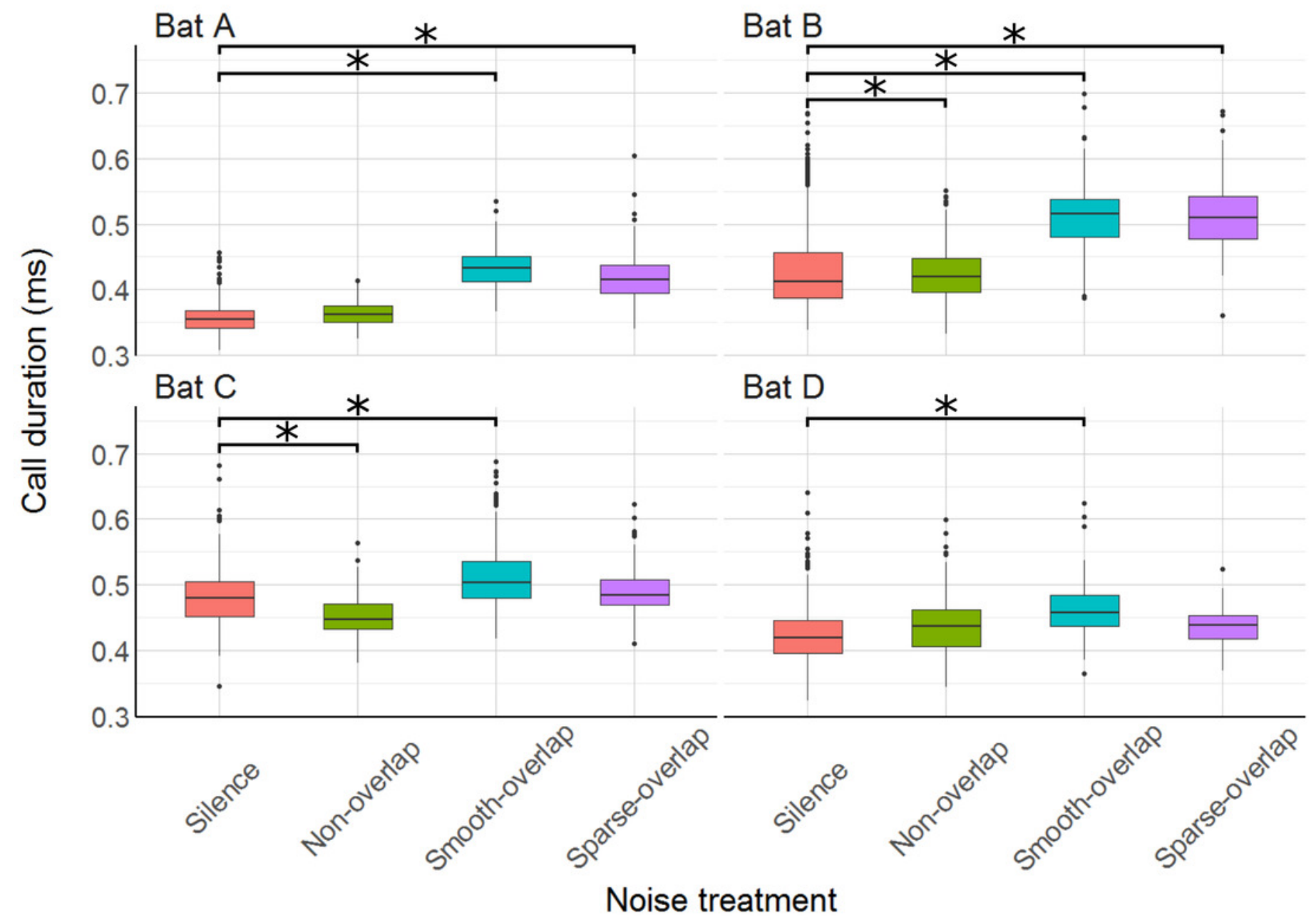




\section{Figure 7}

Relative sound pressure level of echolocation calls during the discrimination tasks by noise treatments.

All four bats significantly increased call level under both smooth- and sparse-overlapping noise. Only bat A also increased call level under smooth non-overlapping noise, and this change was much smaller. Box plots show median (solid line) and first and third quartiles (edges of box); whiskers extend to the rest of the data minus outliers (more than 1.5 times the interquartile range), which are shown as points. Noise treatments have been abbreviated here (as compared to the text) to reduce visual clutter (smooth non-overlapping noise $=$ "Non-overlap"; smooth-overlapping noise = "Smooth-overlap"; sparse-overlapping noise = "Sparse-overlap"). Asterisks denote significant differences $(p<0.05)$ relative to the silence control. 


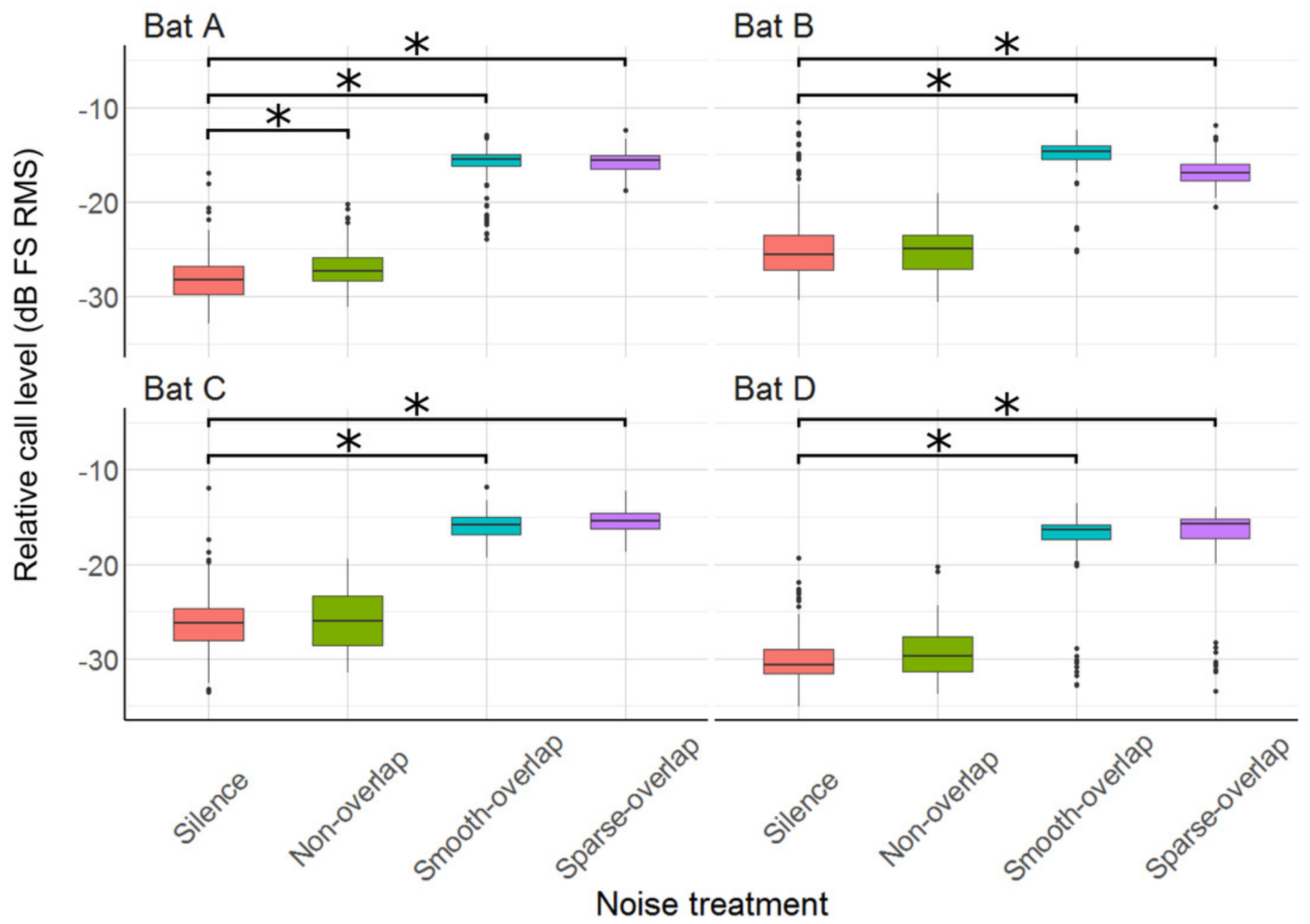




\section{Figure 8}

Peak frequency of echolocation calls during the discrimination tasks by noise treatment.

Peak frequency of bat A increased under smooth non-overlapping noise and decreased under smooth overlapping noise. Peak frequency of bat $\mathrm{C}$ increased under sparse-overlapping noise. Box plots show median (solid line) and first and third quartiles (edges of box); whiskers extend to the rest of the data minus outliers (more than 1.5 times the interquartile range), which are shown as points. Noise treatments have been abbreviated here (as compared to the text) to reduce visual clutter (smooth non-overlapping noise = "Non-overlap" here; smooth-overlapping noise = "Smooth-overlap" here; sparse-overlapping noise = "Sparseoverlap" here). Asterisks denote significant differences $(p<0.05)$ relative to the control treatment. 


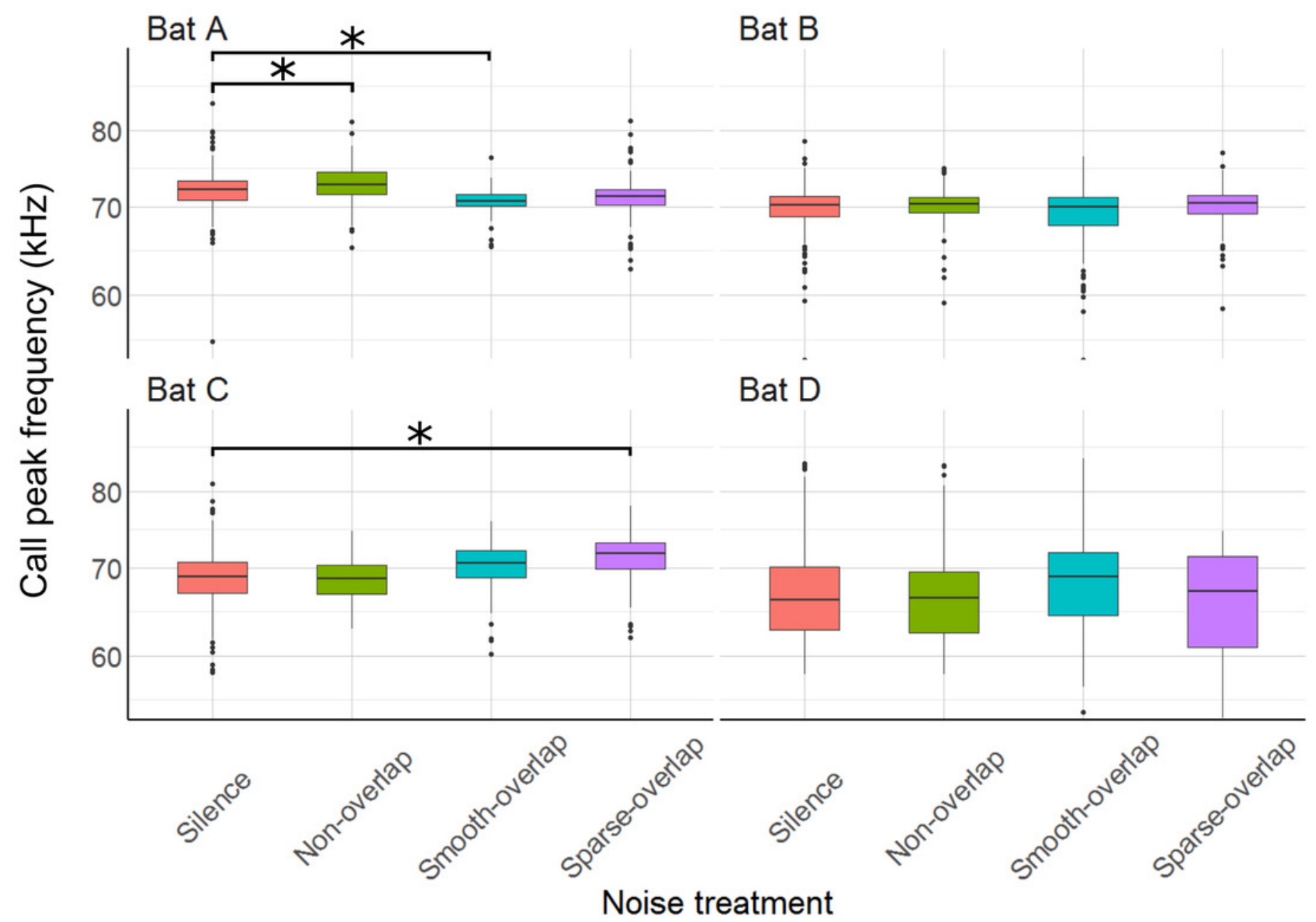




\section{Table $\mathbf{1}$ (on next page)}

Results from generalized linear models for discrimination performance in various noise treatments.

Model results show the estimated differences in discrimination performance (relative to control trials) for the three noise treatments, ripple height, the number of days the bat was in the experiment, and the interaction between each noise treatment and ripple height (i.e. the shape of each performance curve as a function of ripple height), separately for each bat. Data were analyzed with binomial distribution and logit link function. Noise treatments have been abbreviated here (as compared to the text) to reduce visual clutter (smooth nonoverlapping noise = "Non-overlap"; smooth-overlapping noise = "Smooth-overlap"; sparseoverlapping noise = "Sparse-overlap"). 


\begin{tabular}{|c|c|c|c|c|c|}
\hline Bat & Variable & Estimate & SE & $Z$ value & $p$ value \\
\hline A & (Intercept) & -0.766 & 0.275 & -2.791 & 0.02 \\
\hline A & Non-overlap & 0.228 & 0.521 & 0.438 & 0.987 \\
\hline A & Smooth-overlap & 0.317 & 0.423 & 0.749 & 0.911 \\
\hline A & Sparse-overlap & 0.544 & 0.447 & 1.216 & 0.637 \\
\hline A & Ripple height & 0.294 & 0.047 & 6.222 & $<0.001$ \\
\hline A & Day of experiment & 0.110 & 0.095 & 1.165 & 0.673 \\
\hline A & Non-overlap:Ripple height & 0.079 & 0.099 & 0.798 & 0.891 \\
\hline A & Smooth-overlap:Ripple height & -0.096 & 0.064 & -1.504 & 0.435 \\
\hline A & Sparse-overlap:Ripple height & -0.088 & 0.069 & -1.265 & 0.603 \\
\hline B & (Intercept) & -0.544 & 0.243 & -2.240 & 0.096 \\
\hline B & Non-overlap & 0.200 & 0.412 & 0.486 & 0.981 \\
\hline B & Smooth-overlap & 0.441 & 0.377 & 1.171 & 0.668 \\
\hline B & Sparse-overlap & 0.490 & 0.364 & 1.346 & 0.543 \\
\hline B & Ripple height & 0.216 & 0.036 & 6.082 & $<0.001$ \\
\hline B & Day of experiment & 0.003 & 0.107 & 0.025 & 1 \\
\hline B & Non-overlap:Ripple height & -0.012 & 0.059 & -0.209 & 0.999 \\
\hline B & Smooth-overlap:Ripple height & -0.091 & 0.048 & -1.905 & 0.209 \\
\hline B & Sparse-overlap:Ripple height & -0.109 & 0.045 & -2.405 & 0.062 \\
\hline C & (Intercept) & -0.130 & 0.200 & -0.649 & 0.945 \\
\hline C & Non-overlap & 0.277 & 0.312 & 0.888 & 0.847 \\
\hline C & Smooth-overlap & 0.228 & 0.305 & 0.745 & 0.912 \\
\hline C & Sparse-overlap & 0.433 & 0.298 & 1.451 & 0.471 \\
\hline C & Ripple height & 0.118 & 0.022 & 5.465 & $<0.001$ \\
\hline C & Day of experiment & -0.071 & 0.087 & -0.817 & 0.882 \\
\hline C & Non-overlap:Ripple height & -0.071 & 0.028 & -2.518 & 0.047 \\
\hline C & Smooth-overlap:Ripple height & -0.085 & 0.027 & -3.157 & 0.008 \\
\hline C & Sparse-overlap:Ripple height & -0.100 & 0.027 & -3.726 & $<0.001$ \\
\hline $\mathrm{D}$ & (Intercept) & -0.069 & 0.177 & -0.387 & 0.992 \\
\hline D & Non-overlap & -0.486 & 0.327 & -1.487 & 0.445 \\
\hline D & Smooth-overlap & 0.118 & 0.278 & 0.422 & 0.989 \\
\hline $\mathrm{D}$ & Sparse-overlap & 0.280 & 0.283 & 0.990 & 0.789 \\
\hline D & Ripple height & 0.074 & 0.015 & 4.937 & $<0.001$ \\
\hline D & Day of experiment & 0.085 & 0.076 & 1.129 & 0.699 \\
\hline D & Non-overlap:Ripple height & 0.060 & 0.033 & 1.832 & 0.242 \\
\hline D & Smooth-overlap:Ripple height & -0.066 & 0.021 & -3.179 & 0.004 \\
\hline D & Sparse-overlap:Ripple height & -0.066 & 0.021 & -3.141 & 0.008 \\
\hline
\end{tabular}




\section{Table 2 (on next page)}

Threshold of the discrimination performance for ripple detection.

The threshold is the ripple height where bats exceeded a 0.7 probability of a correct choice. For each bat, 1000 simulated discrimination thresholds were generated with a binomial generalized linear model. The lower 0.025 and upper 0.975 percentiles of those data give lower and upper values of the $95 \%$ confidence intervals. Noise treatments have been abbreviated here (as compared to the text) to reduce visual clutter (smooth non-overlapping noise = "Non-overlap"; smooth-overlapping noise = "Smooth-overlap"; sparse-overlapping noise $=$ "Sparse-overlap"). 


\begin{tabular}{|cccccc}
\hline Noise & $\boldsymbol{p}$ & Threshold & Bat & Lower 95\% Cl & Upper 95\% Cl \\
\hline Silent (Control) & 0.7 & 7.05 & A & 5.57 & 8.37 \\
\hline Silent (Control) & 0.7 & 5.39 & B & 4.42 & 6.41 \\
\hline Silent (Control) & 0.7 & 7.58 & C & 5.47 & 9.81 \\
\hline Silent (Control) & 0.7 & 11.42 & D & 8.02 & 15.14 \\
\hline Non-overlap & 0.7 & 5.83 & A & 4.05 & 7.49 \\
\hline Non-overlap & 0.7 & 3.49 & B & 2.44 & 4.63 \\
\hline Non-overlap & 0.7 & 11.62 & $\mathrm{C}$ & 6.16 & 25.07 \\
\hline Non-overlap & 0.7 & 12.14 & D & 9.37 & 14.98 \\
\hline Smooth-overlap & 0.7 & 6.91 & A & 4.59 & 9.15 \\
\hline Smooth-overlap & 0.7 & 6.60 & B & 4.81 & 8.38 \\
\hline Smooth-overlap & 0.7 & 22.92 & C & 11.77 & 702.42 \\
\hline Smooth-overlap & 0.7 & 621.33 & D & 25.87 & $>100000$ \\
\hline Sparse-overlap & 0.7 & 7.63 & A & 4.97 & 10.81 \\
\hline Sparse-overlap & 0.7 & 4.54 & B & 2.85 & 5.95 \\
\hline Sparse-overlap & 0.7 & 28.40 & C & 10.32 & $>100000$ \\
\hline Sparse-overlap & 0.7 & 4395.17 & D & 18.24 & $>100000$
\end{tabular}

1 